\title{
The distortion of distant galaxy images by large-scale structure
}

\author{
R. D. Blandford, ${ }^{1}$ A. B. Saust, ${ }^{1}$ T. G. Brainerd ${ }^{2}$ and J. V. Villumsen ${ }^{2}$ \\ ${ }^{1}$ Theoretical Astrophysics 130-33, California Institute of Technology, Pasadena, CA 91125, USA \\ ${ }^{2}$ Department of Astronomy, The Ohio State University, $174 \mathrm{~W} 18$ th Ave, Columbus, OH 43210, USA
}

Accepted 1991 April 12. Received 1991 April 8; in original form 1991 January 25

\begin{abstract}
SUMMARY
Inhomogeneity in the distribution of mass in the Universe on scales $\$ 100 \mathrm{Mpc}$ can generate a coherent shear distortion or polarization of the images of background galaxies. This distortion may be measurable over patches of the sky up to a few square degrees in size. If this distortion is measured, or conversely, if its magnitude is limited, it should help us understand the degree to which luminosity traces the underlying mass over cosmological scales. A prescription is given for quantifying the galaxy distortion and a propagation equation for its evolution in an inhomogeneous universe is derived. The creation of shear by inhomogeneity is illustrated using model kinematic universes comprising random distributions of point masses, spheres and circular discs designed to simulate the superclusters, voids and 'walls' reported in galaxy velocity surveys. Using these simulations, we estimate that an rms induced ellipticity of $|p|_{\mathrm{rms}} \sim 0.2 \Omega_{\mathrm{LSS}}$ (where $\Omega_{\mathrm{LSS}}$ is the fraction of the mass of the universe clustered on the large scale) will be produced. The angular correlation length is $\sim 1.6^{\circ}$.

In an alternative prescription, the universe is modelled using a power spectrum of density fluctuations and the mean correlation function is computed both analytically and numerically. In these simulations we find that $|p|_{\mathrm{rms}} \sim 0.02$ for biased cold dark matter models of an Einstein-De Sitter universe, and the effective correlation length is $\theta_{1 / 2} \sim 0.5^{\circ}$. For a hot dark matter dominated universe the correlation length is increased to $\theta_{1 / 2} \sim 0.7^{\circ}$.

The faint, blue galaxies discovered by Tyson and collaborators have a surface density of $\sim 3 \times 10^{5} \mathrm{deg}^{-2}$ and should provide an ideal set of sources for measuring this effect.
\end{abstract}

\section{INTRODUCTION}

Recent deep observations of galaxy clusters have revealed the presence of faint blue arcs around galaxy clusters, the best known example being the arcs around Abell 370 (Soucail et al. 1987; Pelló-Descayre et al. 1988; Fort et al. 1988). It is believed that the arcs are gravitationally distorted images of faint distant galaxies, where the cluster acts as a gravitational lens (see e.g. Soucail $e t$ al. 1988; Lynds \& Petrosian 1989; Grossman \& Narayan 1988). Furthermore, Tyson et al. (1984) have developed new observational techniques so that a large number of faint galaxies can be observed and their distortion measured. Their observations show systematic alignments of faint background galaxy images centred on rich foreground galaxy clusters (Tyson, Valdes \& Wenk 1990). They argue that these galaxies are at high redshift $(z \geq 1)$ and that by measuring their distortion, it is possible to probe the mass distribution of the intervening cluster.

In a separate development, Geller \& Huchra (1989) and others cited therein have demonstrated that the distribution of galaxies within the nearby universe has a cellular form with significant overdensities being found in large coherent walls bounding giant $\left(\sim 60 h^{-1} \mathrm{Mpc}\right.$ ) voids (where $h$ is the Hubble constant measured in units of $\left.100 \mathrm{~km} \mathrm{~s}^{-1} \mathrm{Mpc}^{-1}\right)$. Additional evidence for inhomogeneity in the galaxy distribution on these large scales has recently been presented by Broadhurst $e t$ al. (1990) who report periodic structure in pencil beam surveys with wavelength $\sim 128 \mathrm{~h}^{-1} \mathrm{Mpc}$. This is conservatively regarded as further evidence for large-scale structure in the galaxy distribution. More directly, the galaxy-galaxy correlation function measured by Maddox et al. (1990) displays significantly more power on large scales than anticipated (cf. also Efstathiou et al. 1990). 
Bahcall \& Soneira (1984) have presented evidence that Abell Clusters are more clustered than galaxies. Recently, the QDOT survey of IRAS galaxies, Saunders et al. (1991), "indicates there is more structure on large scales than indicated by the standard cold dark matter theory of galaxy formation".

A central question for physical cosmology is the extent to which the light traces the underlying mass. The best evidence for large-scale mass inhomogeneity is dynamical (e.g. Lynden-Bell et al. 1988) and points to the presence of 'great attractors'. However, this evidence remains somewhat controversial due to its sensitivity to small errors in the empirical distance indicators ! used. In this paper, we analyse an alternative method of probing large-scale structure in the Universe using the motions of photons instead of galaxies as tracers of the gravitational field.

Our starting point is the hypothesis that the majority of the faint blue objects observed by Tyson (1988) (estimated to have a sky density of $\left.n_{\mathrm{g}} \sim 3 \times 10^{5} \mathrm{deg}^{-2}\right)$ are cosmologically distant. Although these objects appear to have a higher comoving density than local, bright galaxies, they might well be protogalaxies (e.g. Baron \& White 1987). What is important for our purposes is that they can be resolved in CCD images and assigned a rough orientation. Now, it is an immediate corollary of the cosmological principle that the intrinsic source orientations be randomly distributed on the sky, when averaged over a large enough area. In a homogeneous universe their images should also be randomly distributed on the sky. However, in an inhomogeneous universe, the tidal gravitational field of the intervening matter will shear the image shapes roughly tangentially with respect to the mass concentrations. Averaging over many neighbouring images, we should be able to detect a mean image 'polarization' analogous to the polarization of molecules in a dielectric medium in an electric field (Fig. 1). (Note, however, that a quadrupole is induced by the tensor tidal gravitational field as opposed to a net dipole that results from the vector electric field.)

Let us estimate the magnitude of this image polarization. Assume that these density inhomogeneities have an angular size $\sim \theta$ at a redshift $z \sim 0.5-1$ and an associated rms density (measured in units of the critical density) of $\delta \Omega(\theta)$. (We will henceforth use units in which $G=c=H_{0}=1$ and restrict our attention in this paper to an Einstein-de Sitter cosmology.) The density fluctuations can have either sign. Now a single inhomogeneity of mass $M(\theta) \sim \delta \Omega(\theta) \theta^{3}$ will deflect a ray with impact parameter $\theta$ by an angle $\sim M(\theta) / \theta$. The tidal gravitational force therefore induces an ellipticity $\sim M(\theta) / \theta^{2} \sim \delta \Omega(\theta) \theta$. The ray will pass through $\sim \theta^{-1}$ independent inhomogeneities and their induced ellipticities will add stochastically to give a net image ellipticity

$\varepsilon \sim \delta \Omega(\theta) \theta^{1 / 2}$.

This induced ellipticity ought to be correlated over patches of sky of angular size $\sim \theta$.

Turning aside from the observations, it is conventional in cosmogonic theory to postulate the existence of a power spectrum of relative density fluctuations which can be approximated as a power law.

$$
\begin{aligned}
P(k) & =P_{\max }\left(\frac{k}{k_{\max }}\right)^{n}, \quad k \leqslant k_{\max }, \\
& \sim P_{\max }\left(\frac{\theta}{\theta_{\min }}\right)^{-n}, \quad \theta \geq \theta_{\min },
\end{aligned}
$$

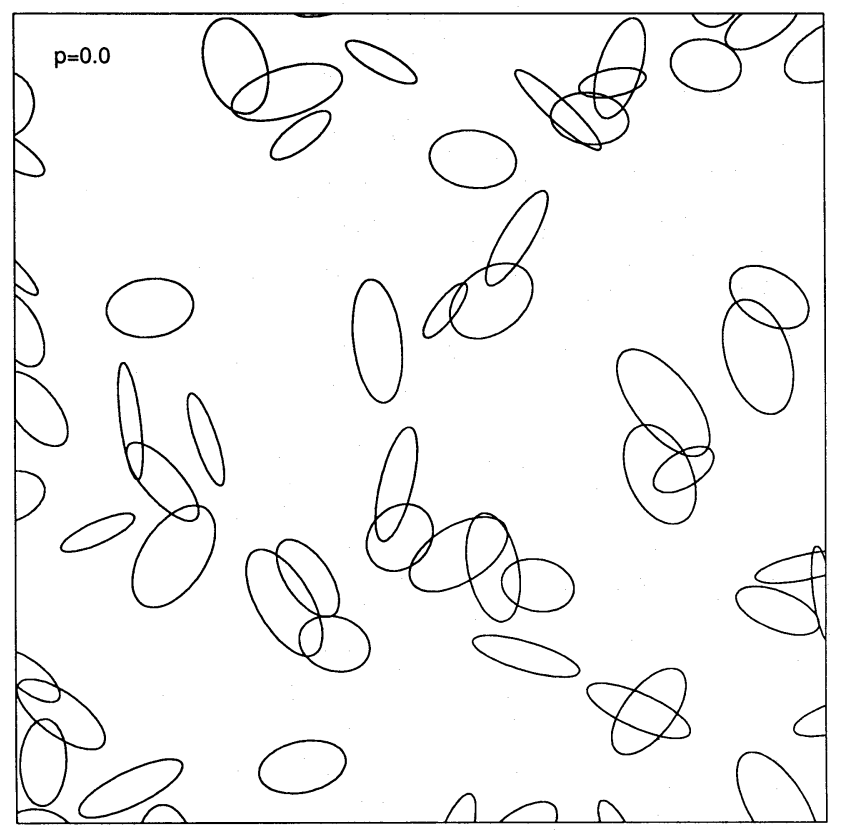

(a)

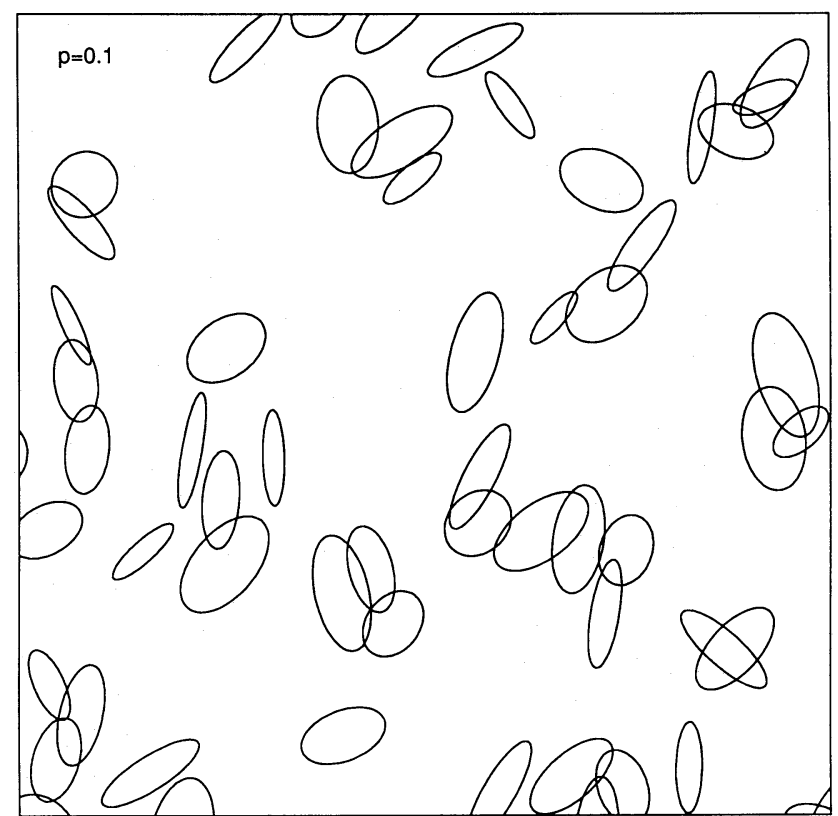

(b)

Figure 1. Polarization of a field of elliptical galaxies. The polarization, $p$, is uniform over the field. (a) $p=0.0(\sim$ no effect $),(\mathrm{b}) p=0.1$. 
where $\theta_{\min } \sim k_{\max }^{-1}$. The density fluctuation on scale $\sim \theta$ then satisfies $\delta \Omega(\theta) \sim P_{\max }^{1 / 2} \theta^{-(3+n) / 2} \theta_{\min }^{n / 2}$ so that the ellipticity induced by fluctuations on this angular scale is

$\varepsilon \sim P_{\max }^{1 / 2} \theta^{-(n+2) / 2} \theta_{\min }^{n / 2}$.

However, if $\theta \gg \theta_{\min }$ we must also consider the stochastic effect of the fluctuations on all smaller angular scales. If we average the ellipticity over an angular scale $\theta \gg \theta_{\min }$ there will be $\sim\left(\theta / \theta_{\min }\right)^{2}$ patches of size $\sim \theta_{\min }$ each contributing a mean ellipticity $\sim P_{\max }^{1 / 2} \theta_{\min }^{-1}$. The measured mean ellipticity on the scale $\theta$ associated with angular scale $\theta_{\min }$ is then anticipated to have the smaller value $\sim P_{\max }^{1 / 2} \theta^{-1}$. This exceeds the ellipticity directly associated with angular scale $\theta$ if $n>0$.

It is not possible to measure this distortion in an individual galaxy image for which the intrinsic orientation is unknown. However, this can be done by averaging over a large number of galaxies. Let the sky density of galaxies be $n_{\mathrm{g}}$ and suppose that their orientations can be measured over an area $\sim \theta^{2}$. The random error in their mean ellipticity should be

$\delta \varepsilon \sim n_{\mathrm{g}}^{-1 / 2} \theta^{-1}$.

Now, if we model the fluctuations by a set of discrete structures with a single characteristic scale, then from equations ( 1 ) and ( 5 ) the random errors will limit the relative density fluctuations that we can hope to measure to

$\delta \Omega \sim n_{\mathrm{g}}^{-1 / 2} \theta^{-3 / 2}$.

For voids and walls with $\theta \sim 0.05, n_{\mathrm{g}} \sim 10^{9} \mathrm{sr}^{-1}$, the minimum measurable density contrast is $\delta \Omega \sim 3 \times 10^{-3}$ correlated over angles of a few degrees.

If, alternatively, we adopt a cold dark matter (CDM) model, the power spectrum of density fluctuations will have a peak on length scales $\sim 10-20 \mathrm{Mpc}$ corresponding to angles $\sim 1^{\circ}$. On these angular scales, the fluctuation amplitude depends upon the degree of biasing, but is likely to correspond to $\delta \Omega \sim 0.1$. This should give a signal in the correlated image ellipticity of $\delta \Omega \theta^{1 / 2}$ on the order of a few per cent, well in excess of the random error. However, the presence of large-scale power in the fluctuation spectrum will create a correlated ellipticity on larger angular scales that diminishes inversely with $\theta(c f$. equation 5 ). Similar remarks apply to hot dark matter (HDM) models with the difference that the correlation length is expected to be larger. On the basis of these estimates, we conclude that it should be possible to measure density fluctuations of magnitudes comparable with those popularly anticipated to be present on large scales in the universe by measuring the shapes of up to a million galaxies. Fortunately this requirement is well matched to the capabilities of newly developed large CCDs.

There is a long history of attempts to extract cosmological data from the shapes of distant images. Kristian (1967) who called this the cosmological distortion effect sought evidence for it in six nearby clusters. Blandford \& Jaroszyński (1981), following Gunn (1967a), (cf. Tomita \& Watanabe 1989) computed the effect of inhomogeneously distributed mass on the images of distant radio sources and showed that, although individual galaxies might produce occasional kinks in intrinsically straight jets and colinear triple sources, the observed evolution in the morphological distortion of radio sources was unlikely to be attributable to the effects of intervening large-scale structure.

Valdes, Tyson \& Jarvis (1983) carried out a photographic survey to $\sim 23$ mag of 40000 galaxies and reported a limit on the cosmologically induced ellipticity of $\sim 0.03$. This was interpreted as limiting the fraction of the critical density clustered on $\sim$ Gpc scales to $\sim 0.1$. Some practical difficulties that would have to be overcome in a more ambitious survey were clearly set out in Tyson (1988).

The specific use of rich clusters of galaxies in bringing about image distortion was discussed by Webster (1985) who argued that this measurement could limit the average mass to light ratio of rich clusters. A positive result has been reported for the individual clusters A1689 and CL1409+ 52 by Tyson et al. (1990) who showed that background blue galaxies are preferentially aligned in the tangential direction with respect to the cluster centre. (More extreme examples of this phenomenon are called arclets or arcs.) Using these observations, Tyson et al. (1984) argued that the dark matter in rich clusters was roughly traced by the luminous galaxies. A major, though not insuperable problem with this technique is that relatively few galaxies are found and the effects of their intrinsic ellipticities are a major source of error in locating the cluster centre and tracing the mass distribution (Kochanek 1990; Miralda-Escudé 1990). The measurement of cluster-induced shear has complementary complications to the present proposal as the distortion is larger but there are relatively few sources involved. Attempts were also made to probe smaller scale structure. Tyson et al. (1984) attempted unsuccessfully to measure the distortion of distant galaxies around foreground galaxies thereby limiting their masses.

More recently, Linder (1990) has analysed the influence of gravitational distortion on microwave background anisotropy showing that if and when fluctuations are detected, they may be enhanced on small scales and diluted on larger scales. (These results are restricted to a Gaussian deflection probability distribution.)

In principle, the cumulative affects of shear can effect angular diameter and luminosity distances (e.g. Watanabe \& Sasaki 1990). Jaroszyński et al. (1990) propagated light rays through CDM universes and find the influence of the shear to be negligibly small in this type of cosmology so that the magnification of distant sources is dominated by Ricci focusing. In addition, they found no instances of multiple imaging induced by larger scale structure than rich galaxy clusters (cf. Babul \& Lee 1990).

The plan of this paper is as follows. In the following section we describe the distortion of a single galaxy image caused by an intervening mass and describe a procedure for measuring it quantitatively by observing a large number of randomly oriented sources. In Section 3 we derive a formalism for propagating image distortion in an inhomogeneous cosmology. In Section 4 we contrast the types of distortion produced by single intervening point masses, truncated isothermal spheres and discs, which we employ to model walls or sheets. We emphasize that discs produce the largest image shear per unit lens mass. This is followed in Section 5 by the numerical calculation of the expected variance and correlation function for the induced image ellipticity in 
universes containing random spatial distributions of the three structures introduced in Section 4 . The most popular and widely discussed type of large-scale structure associates the subluminous mass with cold dark matter assumed to be set down at early epochs with a power-law Gaussian power spectrum whose amplitude is adjusted including a phenomenological biasing factor to account for the present clustering of galaxies and to accommodate constraints on microwave background fluctuations. The nature of the mass distribution is well specified in this cosmogony and in Section 6 we compute the image distortion statistics both analytically and numerically using the results from $N$-body simulations. We then repeat this exercise for universes dominated by hot neutrinos. In the final section, we discuss some of the practical difficulties that may be encountered in attempts to use this technique to measure the distribution of large-scale structure. A preliminary version of these ideas was given in Blandford (1990). Miralda-Escudé (1991) has independently considered many of the questions addressed in this paper.

\section{MODELLING GALAXY DISTORTIONS}

A variety of prescriptions can be used to measure the shape of a galaxy. For our purposes the best procedure is to find a bestfitting ellipse, weighting the measured (pixellated) intensity in some manner. One prescription is to fit a limiting (Holmberg) isophote where the surface brightness equals a fixed fraction of the sky surface brightness. An alternative prescription involves the second moments of the brightness distribution within this isophote (Tyson et al. 1984; Kochanek 1990; Miralda-Escudé 1990) which weights the less elliptical inner regions more heavily but is less susceptible to distortion caused by the shapes of neighbouring galaxies. A general formulation involves computing the matrix

$E_{i j}=\frac{\int d r_{1} d r_{2} f\left[I\left(r_{1}, r_{2}\right)\right]\left(r_{i}-\bar{r}_{i}\right)\left(r_{j}-\bar{r}_{j}\right)}{\int d r_{1} d r_{2} f\left[I\left(r_{1}, r_{2}\right)\right]}$,

where $I\left(r_{1}, r_{2}\right)$ is the intensity at the point with angular coordinates $r_{1}, r_{2}, \bar{r}$ is the galaxy centre defined by

$\bar{r}=\frac{\int d r_{1} d r_{2} f\left[I\left(r_{1}, r_{2}\right)\right] r}{\int d r_{1} d r_{2} f\left[I\left(r_{1}, r_{2}\right)\right]}$

and the function $f(I)$, which will be a delta function in the case that a limiting isophote is fitted, has to be optimized by experiments with real data. This matrix can then be diagonalized so that the eigenvalues, $a^{\prime 2}$ and $b^{\prime 2}$, define the squares of the major and minor axes of the associated ellipse. We can also obtain the position angle of the major axis of the ellipse (north through east) $\phi^{\prime}$. We can characterize the ellipticity and orientation of the ellipse using the single complex number

$\chi^{\prime}=\left(\frac{b^{\prime 2}-a^{\prime 2}}{a^{\prime 2}+b^{\prime 2}}\right) \mathrm{e}^{2 i \phi^{\prime}}$

We call $\chi^{\prime}$ the (complex) orientation of the source.

Suppose we have a source galaxy with an intrinsic equivalent ellipse defined in this manner and characterized by primed coordinates and an orientation $\chi^{\prime}$. The image that we observe after propagation through an inhomogeneous universe will have an equivalent ellipse (unprimed coordinates) that has been expanded, rotated and sheared by a small amount. If we orient our sky coordinate system so that $r_{1}$ and $r_{1}^{\prime}$ advance in a westerly direction and $r_{2}$ and $r_{2}^{\prime}$ to the north, then the general linear transformation from the image plane on to the source plane can be written

$r_{1}^{\prime}=\left(\kappa_{\mathrm{r}}+\gamma_{\mathrm{r}}\right) r_{1}+\left(\kappa_{\mathrm{i}}+\gamma_{\mathrm{i}}\right) r_{2}, \quad r_{2}^{\prime}=\left(-\kappa_{\mathrm{i}}+\gamma_{\mathrm{i}}\right) r_{1}+\left(\kappa_{\mathrm{r}}-\gamma_{\mathrm{r}}\right) r_{2}$,

where $\kappa_{\mathrm{r}}$ is the expansion which measures the image magnification and $\kappa_{\mathrm{i}}$ measures the rotation. The two quantities $\gamma_{\mathrm{r}}$ and $\gamma_{\mathrm{i}}$ together measure the complex shear. We assume that $\kappa_{\mathrm{i}}, \gamma_{\mathrm{r}}$ and $\gamma_{\mathrm{i}}$ are small and linearize in these quantities [note that we do not assume that $\left(\kappa_{\mathrm{r}}-1\right)$ is small]. We find that the complex orientation of the image is given by

$\chi=\chi^{\prime}+\frac{2 \gamma}{\kappa_{\mathrm{r}}}-\frac{2 i \chi^{\prime} \kappa_{\mathrm{i}}}{\kappa_{\mathrm{r}}}-\frac{2 \chi^{\prime}}{\kappa_{\mathrm{r}}}\left(\chi_{\mathrm{r}}^{\prime} \gamma_{\mathrm{r}}+\chi_{\mathrm{i}}^{\prime} \gamma_{\mathrm{i}}\right)$

Now suppose there is some distribution of $\left|\chi^{\prime}\right|$ and that $\phi^{\prime}$ is intrinsically randomly distributed. We then average over $\phi^{\prime}$ to obtain an estimate of the shear from a measurement of the mean image orientation

$p=\frac{2 \gamma}{\kappa_{\mathrm{r}}}=\left\langle\frac{\chi}{1-\chi \chi^{*} / 2}\right)$

accurate to second order in the orientation. In this formula the brackets \langle\rangle denote an average over an area of the sky that is large enough to contain many galaxy images, but smaller than the size of the large-scale structure being probed. We call $p$ the image polarization, a characteristic of the intervening medium, and by mapping it over the sky we can learn about large-scale structure. The influence of shear on the shape of a circular source is illustrated in Fig. 2. 


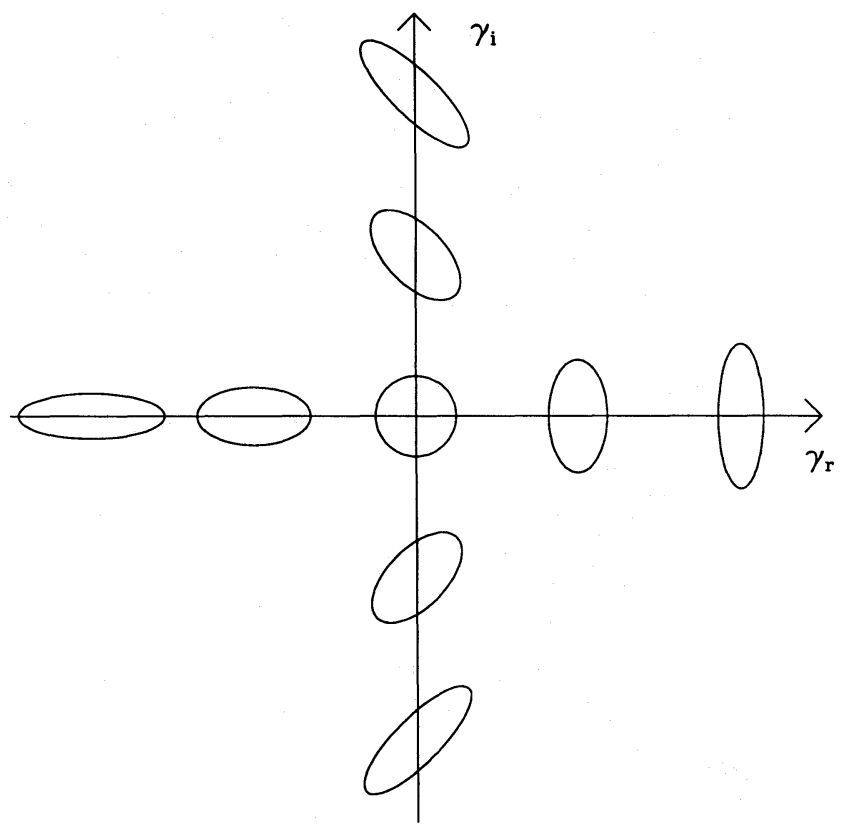

Figure 2. Effect of shear, $\gamma$, on the shape of a circular source. $\kappa$ is set to unity. The real part of $\kappa$ describes a uniform expansion, the imaginary part of $\kappa$ corresponds to a rotation about the origin.

Rewriting equation (12) in terms of the ellipticity, $\varepsilon(=1-b / a)$, we obtain

$p=\left\langle-\varepsilon\left(\frac{1-\frac{3}{2} \varepsilon+\varepsilon^{2}-\frac{1}{4} \varepsilon^{3}}{1-2 \varepsilon+\frac{3}{2} \varepsilon^{2}-\frac{1}{2} \varepsilon^{3}+\frac{1}{8} \varepsilon^{4}}\right) \mathrm{e}^{2 i \phi}\right\rangle$.

When the images are mildly elliptical, as we shall henceforth assume, we need retain only the leading term in equation (13), i.e. $p=\langle\chi\rangle=\left\langle 2 \gamma / \kappa_{\mathrm{r}}\right\rangle=-\left\langle\varepsilon \mathrm{e}^{2 i \phi}\right\rangle$.

Now let us consider how accurately $p$ can be measured and, for the moment, consider only random errors. If there are $N$ randomly distributed galaxies in the sample with rms intrinsic ellipticity, $\varepsilon$, the expectation of the mean polarization of the images when $\gamma=0$ will be

$\langle|p|\rangle=\left\langle\frac{\sum_{i=1}^{N} \chi_{i}}{N}\right\rangle=\varepsilon N^{-1 / 2}$.

For a typical galaxy, $\varepsilon \sim 0.3$ (e.g. an E3 elliptical galaxy or a spiral galaxy inclined at $45^{\circ}$ to the line-of-sight). For an image polarization to be clearly detectable, it must exceed three times this value. In other words, in the absence of systematic errors (seeing, telescope optics etc., which are discussed below) the minimum detectable polarization will be given by

$p \approx 10^{-2}\left(\frac{N}{10^{4}}\right)^{-1 / 2}\left(\frac{\varepsilon}{0.3}\right)$.

We can measure the two-point correlation of $\chi$ by summing over pairs of galaxies. This measurement will be shot-noise limited by the variance of $\chi$ divided by the effective number of pairs of galaxies. The variance of $\chi$ will be the sum of the mean square ellipticity of the galaxies plus the mean square polarization. As long as the rms polarization is less than the rms galaxy eccentricity then a strong small-scale polarization will not degrade the measurement of a weaker polarization induced by largescale structure.

We now turn to the task of evaluating $p$.

\section{PROPAGATION OF DISTORTION IN AN INHOMOGENEOUS UNIVERSE}

The degree of image magnification and polarization depends upon the amplitude and location of departures from spatial inhomogeneity of the mass distribution. We already know from visual inspection of the images of cosmologically distant objects that these effects are not large and it is adequate to restrict our attention to distortions that are linear in the perturbing mass. This limitation circumvents the difficult and controversial question of defining rigorously amplification in a strongly inhomogeneous cosmology and we therefore explicitly exclude rare instances of strong lensing close to galaxies and rich clusters. The propagation of shear is best handled using the optical scalar equation (e.g. Sachs 1961; Penrose 1966; Kristian \& Sachs 1967; Blandford \& Narayan 1986; Dyer \& Oattes 1988; and especially Gunn 1967b). We present a variation on this formalism that is 
well-suited to our application, developing it initially in some generality and then specializing to the case of an Einstein-de Sitter universe.

Consider a small bundle of null geodesics propagating backward in time from the observer toward the source. We require the transverse separation $\xi$ of neighbouring geodesics, measured as a proper distance in the local cosmological rest frame. We designate a fiducial ray and parameterize neighbouring rays using the small angles they make with the fiducial ray at the observer. These angles form a two-dimensional image space which must be mapped on to $\boldsymbol{\xi}$. We parameterize the distance along the ray using the affine parameter $\lambda$. In a weakly perturbed Friedmann-Robertson-Walker Universe,

$\lambda=\int_{t}^{t_{0}} d t^{\prime} a\left(t^{\prime}\right)$,

where $t$ is the cosmic time, $t_{0}$ is the present time and $a(t)$ is the scale factor in the corresponding homogeneous universe [NB $a\left(t_{0}\right)=1$ ]. When $t_{0}-t \ll t_{0}, \lambda$ measures proper distance along the ray. (We continue to set the Hubble constant to unity.) Therefore the angle at the observer that a ray makes with the fiducial ray is simply $\dot{\boldsymbol{\xi}}\left(t_{0}\right)=\dot{\boldsymbol{\xi}}_{0}$.

It is convenient to introduce a Cartesian coordinate basis and write the components of the vector $\boldsymbol{\xi}(\lambda)$ as a complex number $\xi=\xi_{1}+i \xi_{2}$. A general linear map from $\dot{\xi}_{0}$ on to $\xi$ can then be written in the form

$\xi=D_{1}^{*} \dot{\xi}_{0}+D_{2} \dot{\xi}_{0}^{*}$

where the two complex numbers $D_{1}$ and $D_{2}$ can be considered as the two complex components of a vector which we call the Distance Polar, D. (This quantity is similar to a spinar but has different and simpler transformation properties.) When the basis vectors $e_{1}$ and $e_{2}$ on the sky are rotated through an angle $\alpha, D_{1} \rightarrow D_{1}$ and $D_{2} \rightarrow e^{2 i \alpha} D_{2}$. D contains full information about the linearized image distortions. In a homogeneous universe, $D_{1}$ is real and equal to the usual angular diameter distance and $D_{2}$, which describes the shear, vanishes. In an inhomogeneous universe we can compare equation (17) with equation (10) to make the identifications

$\mathrm{D}_{1}=D_{\mathrm{OS}} \kappa \quad \mathrm{D}_{2}=D_{\mathrm{OS}} \gamma$

where $D_{\mathrm{OS}}$ is the angular diameter distance in the smooth universe. For a single scatterer, $\kappa$ will be real. However, in the presence of multiple scattering, $\kappa$ will, in general, be complex, its imaginary part measuring the rotation of the image. Both components of D will likewise be complex numbers in general.

We can now derive a propagation equation for $\mathbf{D}$. First we define the rate of the expansion of the rays in the congruence $\vartheta$ and the (complex) rate of shear, $\sigma$, according to

$\dot{\xi}=\vartheta \xi+\sigma \xi^{*}$.

These definitions are in agreement with the usual relativistic definitions (e.g. Sachs 1961) when evaluated in the local cosmological rest frame. Next we differentiate equations (18) and substitute equation (19) to obtain

$\dot{\mathbf{D}}=\mathbf{S D}$,

where the rate of strain matrix $\mathbf{S}$ is given by

$\mathbf{S}=\left(\begin{array}{c}\vartheta \sigma^{*} \\ \sigma \vartheta\end{array}\right)$

We then invoke the optical scalar equations which describe the evolution of $\vartheta$ and $\sigma$ (e.g. Sachs 1961)

$\dot{\vartheta}+\vartheta^{2}+\sigma \bar{\sigma}=\mathscr{R}=\frac{1}{2} R_{a b} k^{a} k^{b}$

$\dot{\sigma}+2 \vartheta \sigma=\mathscr{F}=C_{a i b j} k^{a} k^{b}\left(t^{i}\right)^{*}\left(t^{j}\right)^{*}$,

where $R_{a b}$ is the Ricci tensor, $C_{a i b j}$ is the Weyl tensor, and $t^{i}$ is a complex null vector parallel propagated along the congruence. The Weyl term represents focusing and distortion by clumps of matter near to, but outside the congruence and the Ricci term represents focusing by matter in the beam.

It is easiest to evaluate $\mathscr{R}$ and $\mathscr{F}$ locally using the local Newtonian gravitational potential, $\Phi$. (We exclude the possible presence of domain walls, strings etc.) In terms of $\Phi, \mathscr{R}$ and $\mathscr{F}$ can be written

$\mathscr{R}=-a^{-2}\left(\Phi_{, 11}+\Phi_{, 22}\right)$
$\mathscr{F}=-a^{-2}\left(\Phi_{, 11}-\Phi_{, 22}+2 i \Phi_{, 12}\right)$,

where the differentiations are carried out with respect to proper distance. By differentiating equation (20), and substituting equations (22) and (23), we obtain a second-order evolution equation for the distance polar

$\ddot{\mathbf{D}}=\mathbf{T D}$,

where the tidal matrix $\mathbf{T}$ is given by

$\mathbf{T}=\left(\begin{array}{cc}\mathscr{R} & \mathscr{F}^{*} \\ \mathscr{F} & \mathscr{R}\end{array}\right)$. 
Equation (26), which is considerably simpler than the usual formulation, can be used to propagate a null geodesic congruence from the observer backwards in time through a weakly inhomogeneous cosmology to a resolved source. It explicitly shows how the tidal gravitational force resolved transverse to the fiducial ray focuses and deforms the congruence of rays around it. Under normal cosmological circumstances, the Ricci term in the tidal matrix and on the right-hand side of equation (24) can be augmented by a term $-a^{-2} \Phi_{, 33}$, involving derivatives along the fiducial ray. This is because the effect of the gravitational force exerted by some element of mass resolved along the ray averages to zero to the order to which we are working. The Ricci term can therefore be rewritten as

$\mathscr{R}=-4 \pi \rho a^{-2}$,

where $\rho$ is the local matter density (assumed to be non-relativistic).

For illustration, let us compute the distance polar in a homogeneous Einstein-de Sitter universe with $t_{0}=2 / 3, \rho=3 / 8 \pi a^{3}$, $a=(3 t / 2)^{2 / 3}$ and $\lambda=2\left(1-a^{5 / 2}\right) / 5$. The rate of shear, $\sigma$, vanishes identically. Solving equation (22), we obtain an expression for the rate of expansion:

$\vartheta=\frac{3 a^{1 / 2}-2}{2 a^{5 / 2}\left(1-a^{1 / 2}\right)}$.

Substituting into equation (20), we obtain the familiar expression for the angular-diameter distance:

$\mathrm{D}_{1}=2 a\left(1-a^{1 / 2}\right)$

More generally, solving equation (26), we can convert the distance polar D into the two observable quantities introduced in the previous section, the mean image magnification fluctuation (relative to a homogeneous universe),

$\Delta M=2\left(1-\frac{\mathrm{D}_{1 r}}{\mathrm{D}_{\mathrm{OS}}}\right)$

(where $r$ denotes the real part) and the mean image polarization (in the small ellipticity limit),

$p=2 \frac{\mathrm{D}_{2}}{\mathrm{D}_{\mathrm{OS}}}$

It will prove useful to work with comoving coordinates $\boldsymbol{x} \equiv\left(x_{1}, x_{2}, x_{3}\right)=2\left(1-a^{1 / 2}\right)\left(\xi_{01}, \dot{\xi}_{02}, 1\right)$. The equation of a null geodesic in these coordinates is simply $x_{1,2}=\xi_{01,2} x_{3}$ (cf. Fig. 3). We emphasize that comoving coordinates can only be used in the smallangle (paraxial) approximation.

\section{IMAGE POLARIZATION FROM INDIVIDUAL DEFLECTORS}

If a density inhomogeneity is interposed between us and a source, it will magnify and distort the image of the source. When the overall size of the source is small compared with the scalelength in the gravitational field, it is conventional to describe this using a magnification tensor whose elements, involving the two complex numbers $\kappa$ and $\gamma$, can be read off from equations (10).

Suppose, for simplicity, that we have a single deflector. We can then define an effective potential $\psi$ by

$\psi=\frac{2 D_{\mathrm{LS}} \Phi^{(2)}}{D_{\mathrm{OL}} D_{\mathrm{OS}}}$

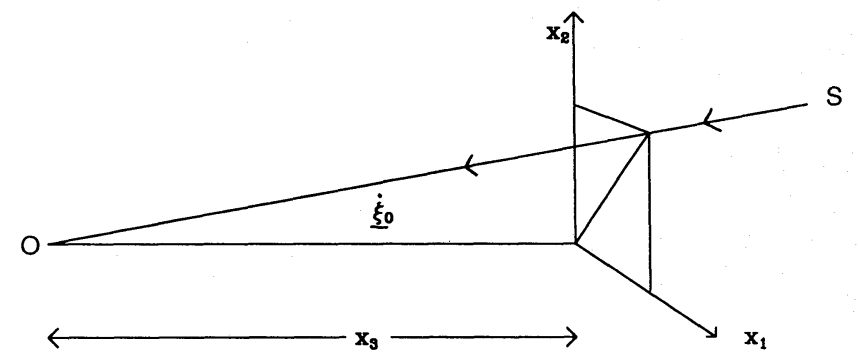

Figure 3. Ray geometry in an Einstein-de Sitter universe. The distance measure is the comoving distance $x_{3}=2\left[1-a^{1 / 2}\right]$, where $a$ is the expansion factor. Comoving distances transverse to the line-of-sight are denoted by $x_{1,2}=\xi_{1,2} / a$. Unperturbed null geodesics are straight lines in this diagram. The small angle $\dot{\boldsymbol{\xi}}_{0}$ is $\left(d x_{1} / d x_{3}, d x_{2} / d x_{3}\right), S$ is the source and $O$ the observer. 
where $\Phi^{(2)}(\boldsymbol{r})$ is the two-dimensional gravitational potential associated with the deflector, $\boldsymbol{r}=\left(r_{1}, r_{2}\right)$ are angular coordinates on the sky and $D_{i j}$, where $i$ and $j$ denote $O b s e r v e r, ~ L e n s$ and Source, are the angular diameter distances (e.g. Blandford \& Narayan 1986). By inspection, we find that

$\kappa=1-\frac{1}{2} \frac{\partial^{2} \psi}{\partial r_{1} \partial r_{1}}-\frac{1}{2} \frac{\partial^{2} \psi}{\partial r_{2} \partial r_{2}}=1-\frac{4 \pi \Sigma D_{\mathrm{OL}} D_{\mathrm{LS}}}{D_{\mathrm{OS}}}$,

$\gamma=-\frac{1}{2} \frac{\partial^{2} \psi}{\partial r_{1} \partial r_{1}}+\frac{1}{2} \frac{\partial^{2} \psi}{\partial r_{2} \partial r_{2}}-i \frac{\partial^{2} \psi}{\partial r_{1} \partial r_{2}}$,

where $\Sigma$ is the surface density of mass. Note that as the gravitational field is curl-free, the imaginary part of $\kappa$ vanishes for a single deflector and there is no rotation of the image. Multiple deflections can, however, create rotation.

As the deflections we are considering are, by assumption, weak, we can linearize the lens equation and evaluate the second derivatives of the potential along the unperturbed trajectories. We are interested in the distribution of $\gamma$ for different lens models. In this section we contrast three possibilities: a point mass, a truncated singular isothermal sphere and a uniform disc.

\subsection{Point mass}

For a circularly symmetric mass distribution, the modulus of the shear can be written in the form

$|\gamma|=-\frac{r}{2} \frac{d}{d r}\left(\frac{1}{r} \frac{d \psi}{d r}\right)$

where we have assumed that the surface density decreases with angular radius $r$ measured from the symmetry axis. For a point mass $M, \psi=K \ln r$, where $K=4 M D_{\mathrm{LS}} / D_{\mathrm{OL}} D_{\mathrm{OS}}$ and the modulus of the image polarization to first order is

$|p| \simeq 2 \frac{|\gamma|}{\kappa}=\frac{2 K}{r^{2}}$,

the integral cross-section for creating polarization with modulus in excess of $|p|$ is

$\sigma(>|p|)=\frac{8 \pi D_{\mathrm{OL}} D_{\mathrm{LS}} M}{D_{\mathrm{OS}}|p|}$

(cf. Fig. 4).

\subsection{Truncated, singular, isothermal sphere}

The potentials of galaxies and clusters of galaxies have been modelled using truncated isothermal spheres in which the mass density diminishes inversely with the square of the radius so that the local internal velocity dispersion remains constant. These

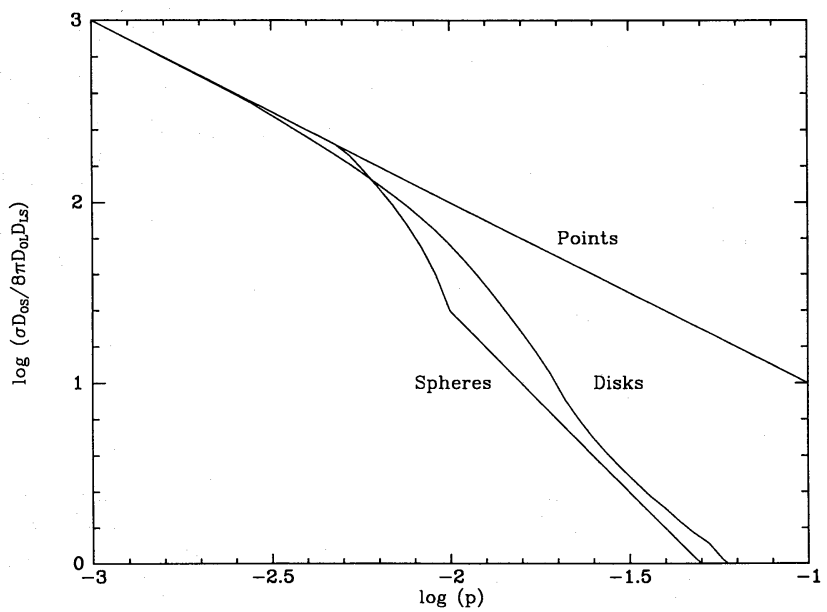

Figure 4. Integral cross-section for production of shear in excess of $|p|$ by point masses, truncated isothermal spheres and uniform, randomly orientated thin discs of similar mass, $M$. The cross-section is measured in units of $4 \pi M D_{\mathrm{OL}} D_{\mathrm{LS}} / D_{\mathrm{Os}}$. The radii of the spheres and the discs are set to $20\left[M D_{\mathrm{LS}} / D_{\mathrm{OL}} D_{\mathrm{OS}}\right]^{1 / 2}$. For small values of the shear, all models behave like point masses. For large values, the disc cross-sections are intermediate between those for points and spheres. 
must be truncated at some (proper) outer radius, $R_{\mathrm{V}}$ in order that the total mass be finite. For simplicity, suppose that the core is vanishingly small. In this case the modulus of the shear is given by equation (37) for $r>R_{\mathrm{V}}$ and

$|p|=\frac{K}{R_{\mathrm{V}} r}$

for $r<R_{\mathrm{V}}$. The integral cross-section is

$\sigma(>|p|)=\pi D_{\mathrm{OL}}^{2}\left[\left(\frac{2 K}{|p|}-R_{\mathrm{V}}^{2}\right) \Theta\left(\frac{2 K}{R_{\mathrm{V}}^{2}}-|p|\right)+\frac{K^{2}}{R_{\mathrm{V}}^{2}|p|^{2}} \Theta\left(|p|-\frac{K}{R_{\mathrm{V}}^{2}}\right)+R_{\mathrm{V}}^{2} \Theta\left(\frac{K}{R_{\mathrm{V}}^{2}}-|p|\right)\right]$,

where $\Theta(x)$ is the step function. $\sigma(>|p|)$ is also shown in Fig. 4. Comparing the result for the point mass with the truncated singular isothermal sphere, we see that a point mass presents a larger cross-section for large shear than an isothermal sphere. This is generally the case: the softer the surface potential, the lower the induced shear. This potential can also be used to model voids if the mass of the sphere is negative. Introducing a core radius into the potential makes only minor changes.

\subsection{Inclined uniform disc}

Our third model inhomogeneity is a uniform circular disc with mass $M$ and radius $R_{\mathrm{D}}$ inclined at an angle $i$ to the line-of-sight. This is a simple model of an individual 'wall' or sheet of matter. In the Appendix, we calculate the components of the surface potential together with its second derivatives. Combining equations (A122) and (A123), we find that

$p=\frac{8 M D_{\mathrm{LS}} \sec i \operatorname{cosec}^{2} i(1-\cos i)^{2}}{R_{\mathrm{D}}^{2} D_{\mathrm{OL}} D_{\mathrm{OS}}}$,

when the ray passes through the projected disc, and

$p=\frac{16 M D_{\mathrm{LS}} \operatorname{cosec}^{2} i}{R_{\mathrm{D}}^{2} D_{\mathrm{OL}} D_{\mathrm{OS}}}\left\{\frac{\left[1-\left(1-\xi_{\mathrm{m}}\right)^{1 / 2}\right]\left[\left(1-\xi_{\mathrm{m}}\right)^{3 / 2} r_{1}^{2}-r_{2}^{2}\right]+i r_{1} r_{2} \zeta_{\mathrm{m}}\left(1-\xi_{\mathrm{m}}\right)^{1 / 2}}{\left(1-\zeta_{\mathrm{m}}\right)^{2} r_{1}^{2}+r_{2}^{2}}\right\}$

where

$\zeta_{\mathrm{m}}=\frac{\left(r_{1}^{2}+r_{2}^{2}+R_{\mathrm{D}}^{2} \sin ^{2} i\right)-\left[\left(r_{1}^{2}+r_{2}^{2}+R_{\mathrm{D}}^{2} \sin ^{2} i\right)^{2}-4 r_{1}^{2} R_{\mathrm{D}}^{2} \sin ^{2} i\right]^{1 / 2}}{2 r_{1}^{2}}$,

when the ray passes outside the disc.

We have also computed the integral cross-section for creating image polarization in excess of $|p|$, averaging over the disc inclination $i$ (Fig. 4). Note that cross-section for creating large shear is larger than that associated with an isothermal sphere of the same mass and radius, but smaller than that of a point mass.

\section{IMAGE DISTORTION CAUSED BY RANDOM DEFLECTORS}

The first type of model universe we consider is one containing a random distribution of simple, purely kinematic model deflectors. Specifically, we consider the three types of deflector we introduced in the preceding section. We suppose that a comoving number density $n_{0}$ of deflectors of mass $M$ with mean cosmological density $\Omega_{M}=8 \pi n_{0} M / 3$ is associated with these deflectors and that $\Omega_{M}$ is sufficiently small that we can ignore the small change in the propagation through the background universe when computing the image polarization. We suppose that the deflectors maintain their comoving size and their masses throughout the expansion of the universe. In this sense, our density inhomogeneity model is strictly kinematic. Relative density fluctuations with $\Omega_{M} \gtrsim 0.1$ on scales $\gtrsim 100 \mathrm{Mpc}$ cannot grow gravitationally without producing unobserved large deviations from the Hubble flow. Of course there will be evolution in both these quantities, however, it turns out that the polarization is dominated by deflectors with redshifts $z \sim 0.3-0.8$ and, on the large scales of interest to us, the mass contained within these individual deflectors cannot have changed appreciably over this redshift interval without there being extremely large, and unobserved, velocities with respect to the Hubble flow.

A visual inspection of faint galaxy (and radio galaxy) fields is sufficient to demonstrate that the images of the majority of cosmologically distant objects are not strongly polarized. Therefore, we can assume that any measurable effect will be at best weak and analysable using a perturbative approach. We handle multiple deflections by evaluating the individual deflections along the unperturbed rays and then summing them. This corresponds to retaining only the linear terms in the multiple-lensing expansion given by Blandford \& Narayan (1986). We are therefore ignoring the few instances when sources lie close to caustics and are strongly magnified or even multiple imaged. These caustics are believed to be formed by smaller mass concentrations than those under investigation here. For the reasons outlined in the introduction, this small-scale structure, associated with the cores of the richest clusters, favourably aligned groups and individual galaxies contributes insufficient power to the overall power spectrum of density fluctuations to give a significant stochastic contribution to the overall signal on large angular scales. 
Nevertheless, we have tested this approximation by integrating the full ray propagation equation (26) along the line-of-sight and producing similar results to those found using the linear approximation.

\subsection{Point masses}

An individual point mass, $M$, located at the origin will induce an image polarization given by equation (37)

$p=\frac{2 \gamma}{\kappa}=\frac{8 M D_{\mathrm{LS}} \mathrm{e}^{2 i \alpha}}{D_{\mathrm{OS}} D_{\mathrm{OL}} r^{2}}$

where $\alpha=\tan ^{-1}\left(r_{2} / r_{1}\right)$. The mean polarization from an isotropic universe is zero. Next consider two rays, labelled $\mathrm{i}$ and $\mathrm{j}$, separated in angle by a distance $\theta$ and passing by the same mass. We form the product

$p_{\mathrm{i}} p_{\mathrm{j}}^{*}=\frac{64 M^{2} D_{\mathrm{LS}}^{2} \mathrm{e}^{-2 i \phi}}{D_{\mathrm{OS}}^{2} D_{\mathrm{OL}}^{2} r_{\mathrm{i}}^{2} r_{\mathrm{j}}^{2}}$,

where $r_{\mathrm{i}}$ and $r_{\mathrm{j}}$ are the angular separations of the rays from the mass and $\phi=\alpha_{\mathrm{j}}-\alpha_{\mathrm{i}}$ is the angle on the sky that the two ray directions subtend at the mass.

Now let $n_{0}$ be the comoving number density of these masses. In an EDS cosmology the number of masses in a volume element referred to ray $i$ is

$d N=n_{0} a^{\prime-3} \cdot r_{\mathrm{i}} d r_{\mathrm{i}} d \alpha_{\mathrm{i}} D_{\mathrm{OL}}^{2} \cdot a^{\prime 1 / 2} d a^{\prime}$.

If individual masses are uncorrelated and we assume that sources $\mathrm{i}$ and $\mathrm{j}$ are at the same redshift, then we can form the twopoint image polarization correlation function by integrating over all masses and averaging over many pairs of directions.

$C_{\mathrm{pp}}(\theta)=\left\langle p_{\mathrm{i}} p_{\mathrm{j}}^{*}\right\rangle_{\theta}=\left\langle\chi_{\mathrm{i}} \chi_{\mathrm{j}}^{*}\right\rangle$,

where $\chi_{\mathrm{i}}$ are the individual image orientations and \langle\rangle$_{\theta}$ denotes an average over all pairs of directions separated by an angle lying within a small interval around $\theta$. We can compute an estimate of the correlation function from

$C_{\mathrm{pp}}(\theta)=\int d N p_{\mathrm{i}} p_{\mathrm{j}}^{*}=\frac{64 M^{2} n_{0}}{D_{\mathrm{OS}}^{2}} \int_{a}^{1} \frac{d a^{\prime} D_{\mathrm{LS}}^{2}}{a^{\prime 5 / 2}} \int_{0}^{\infty} \frac{d r_{\mathrm{i}}}{r_{\mathrm{i}}} \int_{0}^{2 \pi} \frac{d \alpha_{\mathrm{i}}}{r_{\mathrm{j}}^{2}} \cos 2 \phi$,

where $r_{\mathrm{j}}^{2}=r_{\mathrm{i}}^{2}+\theta^{2}-2 r_{\mathrm{i}} \theta \cos (\alpha)$.

Performing the integrals, we eventually obtain

$C_{\mathrm{pp}}(\theta)=\frac{128 \pi n_{0} M^{2}\left(1-a^{1 / 2}\right)}{3 a^{1 / 2} \theta^{2}}$.

Now define $\Omega_{M}=8 \pi n_{0} M / 3$ to be the mean cosmological mass density, then

$C_{\mathrm{pp}}(\theta)=\frac{16 \Omega_{M} M\left(a^{-1 / 2}-1\right)}{\theta^{2}}$.

Numerically we obtain

$$
\begin{aligned}
C_{\mathrm{pp}}(\theta) & =3.1 \times 10^{-3} \Omega_{M} h M_{12}\left[(1+z)^{1 / 2}-1\right]\left(\theta^{\prime}\right)^{-2} \\
& =8.5 \times 10^{-3} \Omega_{M} h M_{16}\left[(1+z)^{1 / 2}-1\right]\left(\theta^{\circ}\right)^{-2},
\end{aligned}
$$

where $M_{n}=M / 10^{n} M_{\odot}, \theta^{\prime}$ is measured in minutes and $\theta^{\circ}$ is measured in degrees. This simple result, although correct, is not useful in practice. The autocorrelation function diverges as $\theta \rightarrow 0$, indicating that the large distortions caused by close encounters dominate the integral and for these the weak distortion approximation is invalid. This is brought out by numerical simulations. We have traced rays through model universes containing random point masses and attempted to form an estimate of the polarization correlation function $C_{\mathrm{pp}}$. Our procedure, which we followed in all subsequent simulations, was to set down point masses randomly within a pyramid with the observer at the vertex and with a base of side 0.2 rad. We then defined a smaller pyramid within this deflector pyramid with base of side $0.1 \mathrm{rad}$ within which we traced rays along random directions. Typically we were interested in masses $\sim 10^{-6}$ in cosmological units $\left(\right.$ or $\left.\sim 10^{16} M_{\odot}\right)$ and of the order of a hundred such masses were used within the deflector pyramid. The complex image polarization $p$ was computed along each of $N_{\mathrm{r}}$ rays summing the contributions from each of the masses. We limited the measured polarization to $|p|<1$, thereby excluding strong distortion. The two point correlation function for the polarization for a ray separation $\theta$ was then estimated by averaging over all the $N_{\mathrm{r}}\left(N_{\mathrm{r}}-1\right) / 2$ pairs of rays, that lay within a bin centred on $\theta$.

For a given realization of the universe, we found that five to ten thousand rays were usually adequate to furnish an acceptable estimate of $C_{\mathrm{pp}}$. Including deflectors outside the deflection pyramid, or changing its shape had an insignificant effect on $C_{\mathrm{pp}}$. 


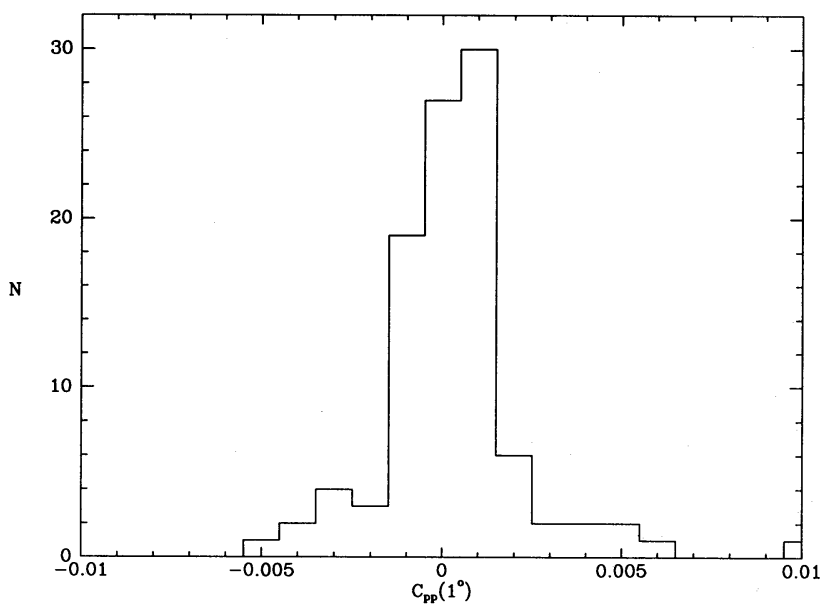

Figure 5. Histogram of the correlation function for sources separated by $1^{\circ}$ in a universe containing a density $\Omega_{M}=0.1$ of point masses, $M=6 \times 10^{-7}\left(4 \times 10^{16} M_{\odot}\right)$.

However, the derived correlation functions fluctuated wildly from realization to realization. A histogram exhibiting the results for $C_{\mathrm{pp}}\left(1^{\circ}\right)$ for one hundred universes is shown in Fig. 5.

Despite the large variance in $C_{\mathrm{pp}}$, its mean value is representative of what we could hope to measure. In our illustrative model we choose $n_{0} \sim 2 \times 10^{4}, \Omega_{M}=0.1$ and $M \sim 6 \times 10^{-7}$ (or $\sim \times 10^{17} M_{\odot}$ ). The two-point correlation function for the image polarization $p$ for an angular separation $\theta$ and an assumed source redshift $z_{\mathrm{s}}=3$ is $C_{\mathrm{pp}}(\theta) \sim 10^{-3}\left(\theta^{\circ}\right)^{-2} \mathrm{deg}^{-2}$. This will scale in proportion to the assumed value of $\Omega$ for large angular scales. There should therefore be a correlated ellipticity $\sim 0.3 \Omega_{M}$ in the image shapes on angular scales of order a degree. However, the distribution of correlations is very broad and of either sign because it is dominated by small impact parameters and so the procedure proposed in this paper is not really suitable for this case. If a significant fraction of the mass density of the universe is contained in individual masses, dense enough to multipleimage background galaxies (for example invisible galaxy clusters) then the best way to detect this will be through observing strong focusing by individual masses rather than by measuring the accumulated effects of many weak deflectors.

\subsection{Voids}

A more realistic model of large-scale structure is obtained if we assume that a fraction of the universe is contained in underdense regions called voids. We treat these as randomly located spheres whose radii, $R_{\mathrm{V}}$ expand with the universe. The density deficit profile is assumed to have the truncated isothermal form of Section 4.2. The fractional underdensity is measured by the density parameter $\Omega_{\mathrm{v}}$, this time as a negative number. If, as observations indicate, the filling factor of these voids is large, $\approx 0.5$, then the incidence of overlapping voids is quite high. Provided the fractional underdensity per void is small, the total density should nowhere become negative.

The radial profile of the void underdensity that we adopt is the truncated, isothermal sphere introduced in Section 4.3. It is necessary to introduce a finite core radius into this potential to prevent the central density from becoming negative. In practice, our results are quite insensitive to the choice of this core radius including the value zero. We set their comoving radii to be $R_{\mathrm{V} 0}=0.02=60 \mathrm{~h}^{-1} \mathrm{Mpc}$, to match the scale of the structures described in the introduction, retaining the same number density $n_{0}=2 \times 10^{4}$ as we used in the case of the point masses so that the void filling factor is $f=4 \pi n_{0} R_{\mathrm{V} 0}^{3} / 3=0.67$. The mean free path for encounters with the denser regions outside the voids is $\ell \sim 1 / \pi R_{\mathrm{v} 0}^{2} n_{0} \sim 0.04$ or $\sim 120 \mathrm{~h}^{-1} \mathrm{Mpc}$.

With this much softer potential we find that the variance in the measured correlation function is greatly reduced. The results are presented in Fig. 6 with the error bars denoting $1 \sigma$ standard errors between different simulations. The rms correlated ellipticity, $\left\langle|p|^{2}\right\rangle^{1 / 2}$, for $z_{\mathrm{s}}=3$ was found to be $0.25 \Omega_{\mathrm{v}}$, somewhat smaller than the equivalent point-deflector value, as expected. The sensitivity of the correlation function to the assumed filling factor at fixed $R_{\mathrm{V} 0}$ and $\Omega_{M}$ is not great.

For reasons that we discuss below, we define the correlation length to be $\theta_{1 / 2}$ where

$\int_{0}^{\theta_{1 / 2}} 2 \pi \theta^{\prime} C_{\mathrm{pp}}\left(\theta^{\prime}\right) d \theta^{\prime} \equiv \frac{1}{2} \int_{0}^{\theta_{0}} 2 \pi \theta^{\prime} C_{\mathrm{pp}}\left(\theta^{\prime}\right) d \theta^{\prime}$

$\theta_{0}$ is the angle at which the correlation function, $C_{\mathrm{pp}}(\theta)$, crosses zero. Adopting this definition, $\theta_{1 / 2}=1.6^{\circ}$. As expected, the correlation length scales with the assumed void size. Naturally, the correlation function is unchanged if we change the sign of the density fluctuations and model density excesses rather than voids. However, the pattern of image deformations observable around an individual void is distinguishable from that surrounding a positive density enhancement. With a void, the images are, on average, elongated in the radial as opposed to the tangential direction with a density excess. 


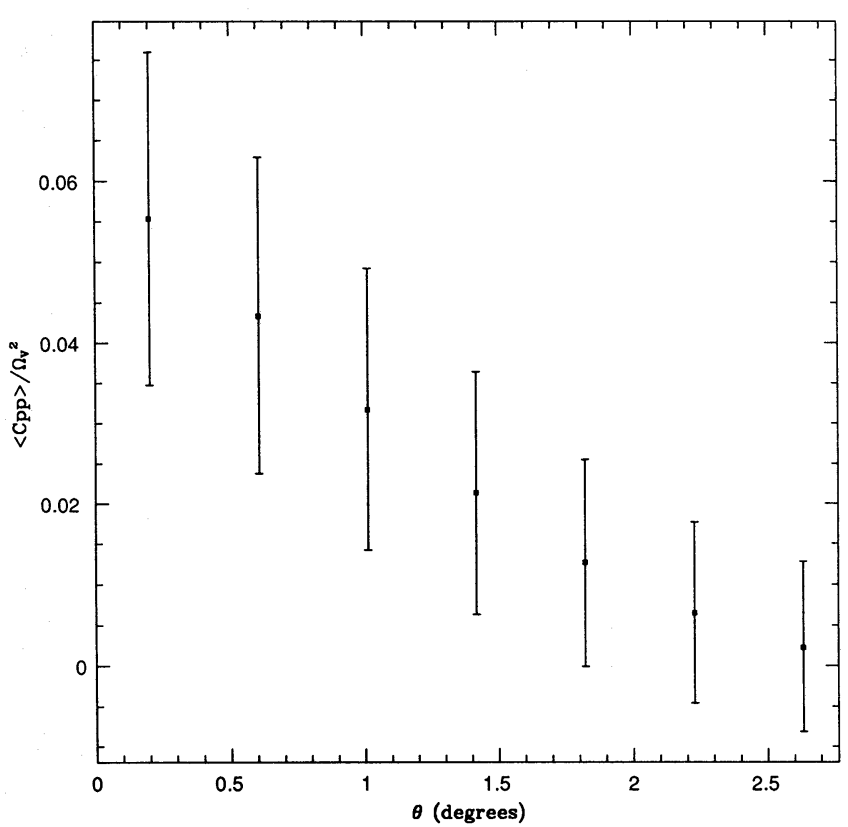

Figure 6. The correlation function, $C_{\mathrm{pp}}$ ( $c f$. equation 47), as a function of source separation, $\theta$, for a universe consisting of uniformly distributed voids (represented by negative mass spheres). The simulations were done with 3000 sources at a redshift of three in a square window of side $0.1 \mathrm{rad}$, the number density of the voids was $2 \times 10^{4}$ and the comoving void radii were 0.02 Hubble lengths. One hundred simulations were performed and the errorbars show the $1-\sigma$ error.

\subsection{Discs}

Our third kinematic model of large-scale structure focuses on the wall or sheet structures. These we model rather primitively (in the absence of a more detailed understanding of their shapes and connectivity) as a random distribution of thin uniform circular discs of radius $R_{\mathrm{D}}$. Again we make no provision for overlap, and assume that $\Omega_{\mathrm{D}} \ll 1$. To facilitate comparison with the preceding two cases, we choose $n_{0}=2 \times 10^{4}$ as before. However, in order to match the mean free path for a line-of-sight to intersect a disc, $\ell=2 / \pi n_{0} R_{\mathrm{D} 0}^{2}$, to the void wall mean free path, we must increase the disc radius to $R_{\mathrm{D} 0}=0.028$.

The two-point correlation function is shown in Fig. 7(a). It is quite similar to that obtained in the void model although the rms correlated ellipticity is slightly smaller $\sim 0.2 \Omega_{\mathrm{D}}$. The correlation length, $\theta_{1 / 2} \sim 1.5^{\circ}$, is essentially unchanged. Again for discs of given size and space density, the correlation function is found to be proportional to $\Omega_{M}^{2}$ and the correlation length increases roughly in proportion to $R_{\mathrm{D}}$.

\subsection{Source redshift distribution}

We have so far assumed that all the sources are at redshift $z_{\mathrm{s}}=3$. The faint blue galaxies are believed to lie mostly between redshifts 1 and 3 (Tyson et al. 1990). We should therefore determine how the correlation function is degraded when the sources are assumed to be located closer to us. We exhibit the disc correlation function for sources at lower redshifts, $z_{\mathrm{s}}=1,2[\mathrm{Fig}$. $7(\mathrm{~b})$ and (c)], as well as for a uniform distribution in comoving volume between $z_{\mathrm{s}}=1$ and $z_{\mathrm{s}}=3[$ Fig. $7(\mathrm{~d})]$. It is found that the correlation function is only seriously degraded when the majority of the sources have $z_{\mathrm{s}} \lessgtr 1$.

\subsection{Position angle differences}

An alternative statistic to the correlation function that can be used to measure the image polarization is the mean (unsigned) position angle differences between images separated by a fixed angle on the sky. If the images were randomly oriented we expect that this quantity $\langle|\Delta \phi|\rangle$ would have a value $\pi / 4$ independent of separation. A smaller value of $\langle|\Delta \phi|\rangle$ corresponds to a net correlation. This statistic is equivalent to the above, however, it has the advantage that it is more reliable to observe the position angle of an image as opposed to observing the complex orientation of the image and it is more useful from an observational point of view. Notice that the correlation function in this case is not proportional to $\Omega_{\mathrm{D}}^{2}$ as it is above (it is the deviation from $\pi / 4$ that is proportional to $\Omega_{\mathrm{D}}^{2}$ ), and a value of $\Omega_{\mathrm{D}}=1$ has been used in these simulations. An example of this statistic for our standard disc-filled universe is shown in Fig. 8.

\section{DISTORTION FROM A POWER SPECTRUM OF DENSITY FLUCTUATIONS}

\subsection{Linear theory}

An alternative method for modelling large-scale structure is to assume that a power spectrum $P(k)$ of density fluctuations is set down at early times and that these fluctuations grow by gravitational instability as the universe expands. It has been shown (e.g. 


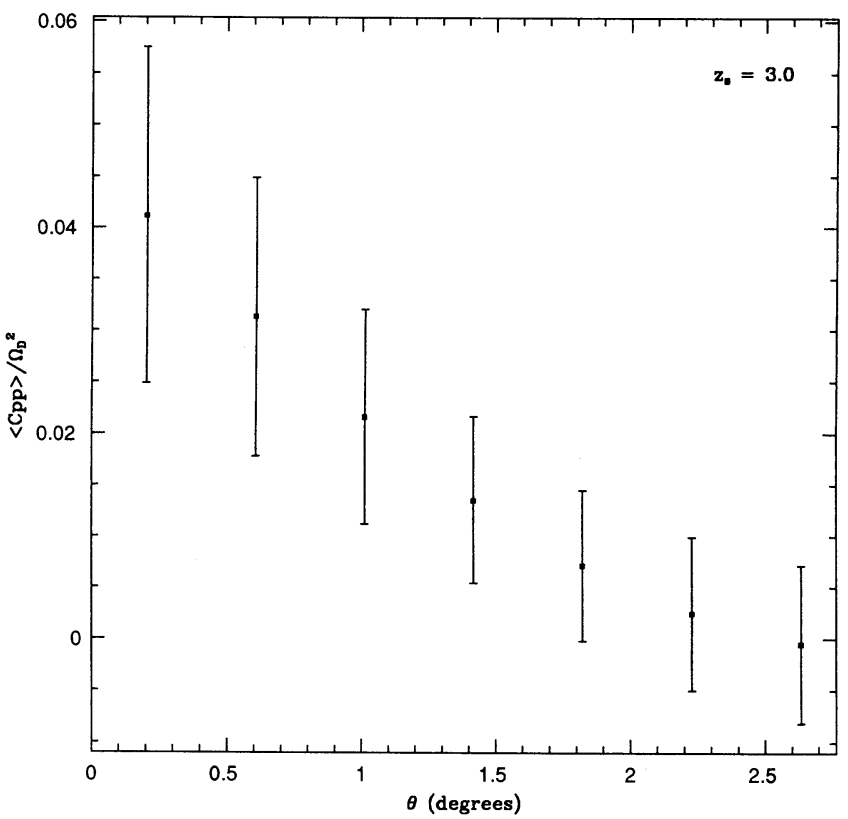

(a)

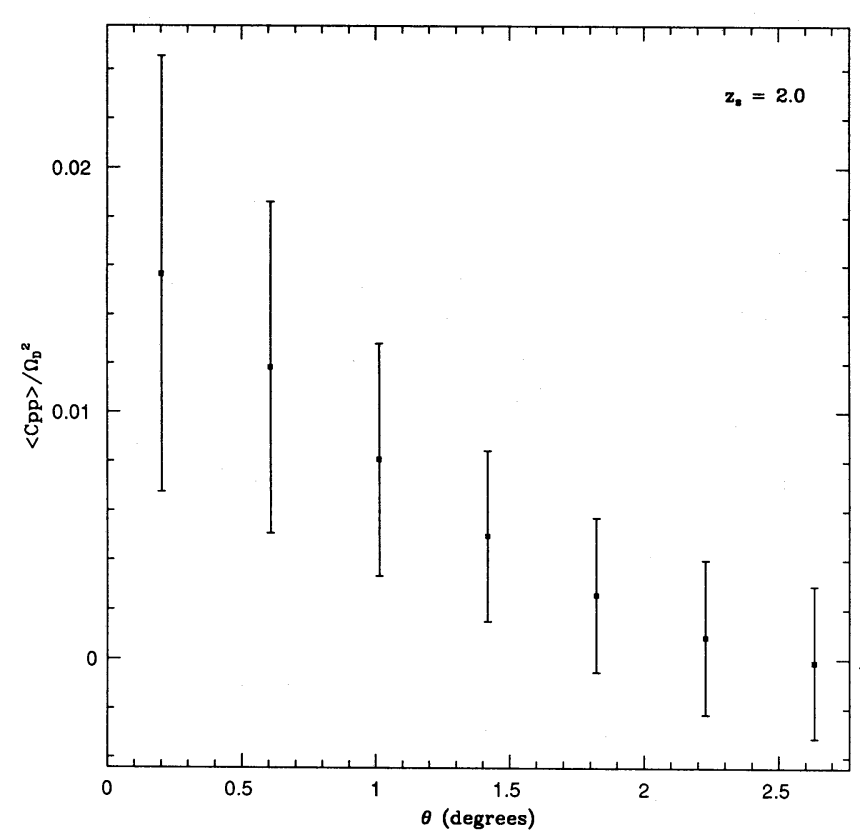

(c)

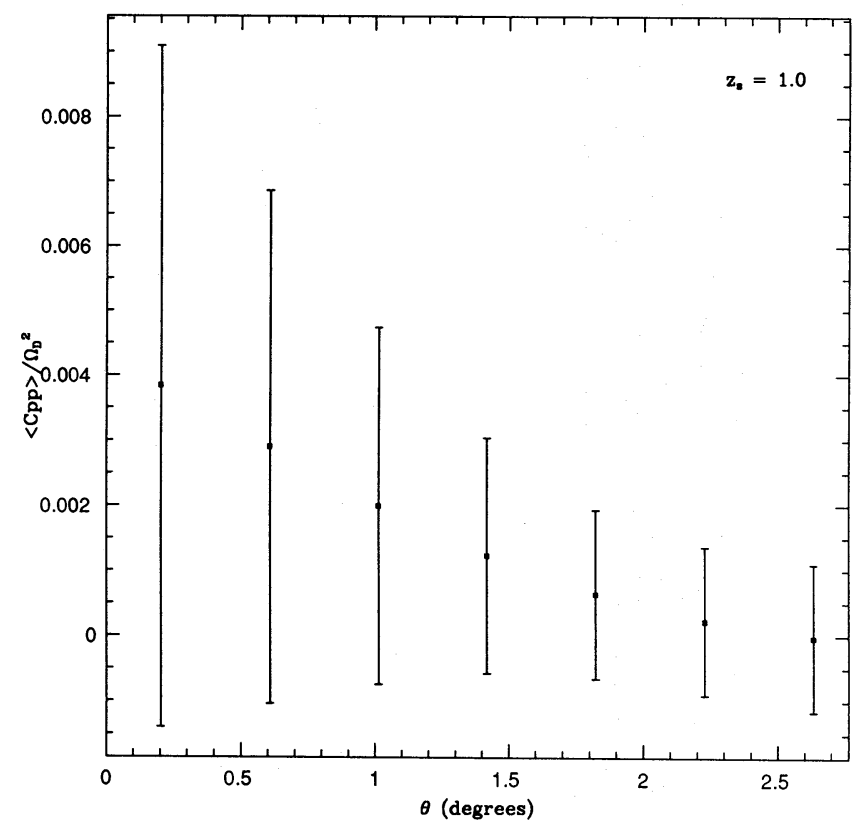

(b)

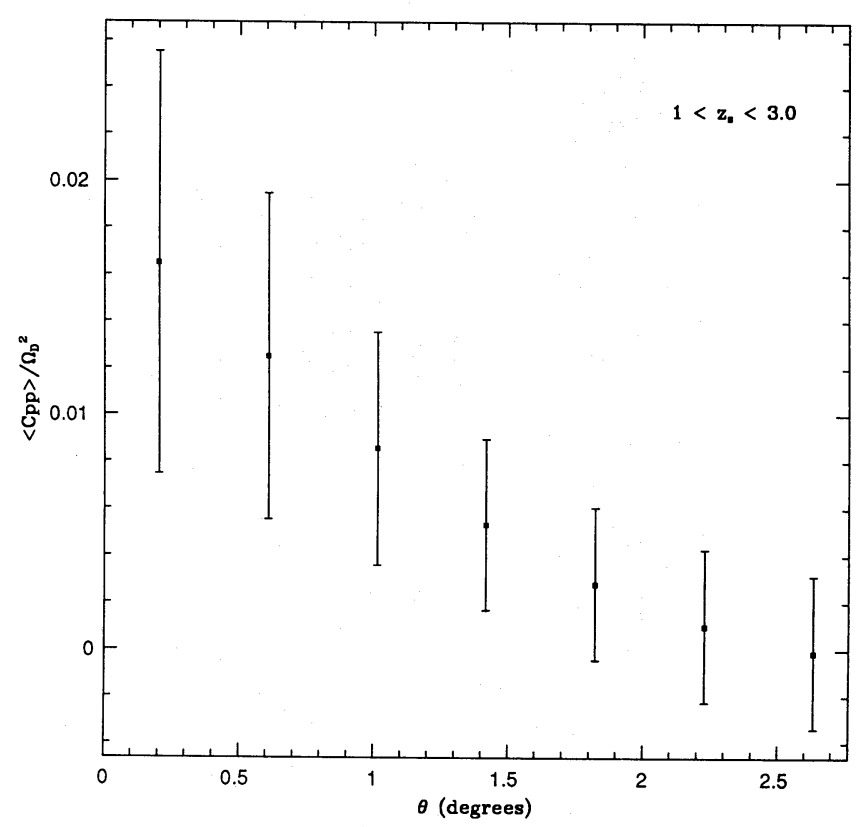

(d)

Figure 7. The correlation function for a universe containing walls (represented by discs). The simulations were performed with the same parameters as in Fig. 6 except that the disc radii were 0.028 Hubble lengths. Furthermore it is shown how the correlation function depends on source redshift. (a) $z_{\mathrm{s}}=3$, same value as in Fig. 6, (b) $z_{\mathrm{s}}=1$, (c) $z_{\mathrm{s}}=2$ and (d) $1<z_{\mathrm{s}}<3$. As can be seen from the figures, the effect is seriously degraded when $z_{\mathrm{s}} \leqslant 2$.

Davis et al. 1985; Park 1990; Villumsen \& Brainerd 1990) that large-scale structure can be simulated under these assumptions, though the extent to which this conforms with observation is still a matter of controversy. In this section, we calculate the expected image magnification and polarization correlation function in model universes based on cold and hot dark matter fluctuation spectra, and we assume $\Omega_{0}=1$.

We assume that the relative density fluctuations, $\delta$, on scales of interest remain small and grow linearly with the expansion factor $a$. As $\delta \ll 1, \kappa$ will vary little from its value in a homogeneous universe with the same mean density. Our calculations are performed in comoving coordinates so $\Phi$ and $\Phi_{, \mathrm{ij}}$ are the potential and its second derivatives with respect to comoving coordinates. In terms of these coordinates the mean density is $3 / 8 \pi$. The subscript zero refers to the present, i.e. $a=1$. 


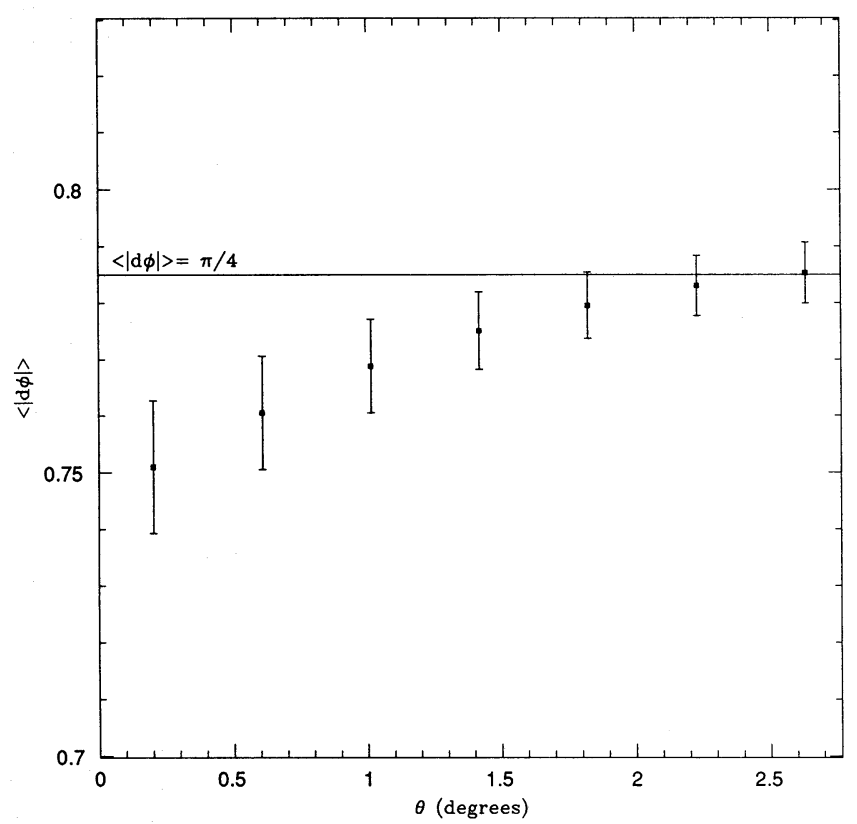

Figure 8. Position angle differences: if the Universe is homogeneous, the mean unsigned position angle difference between sources, $\langle|d \phi|\rangle$, would be $\pi / 4$ independent of $\theta$. Using the same model as in Fig. 7(a) the mean unsigned position angle difference is calculated as a function of $\theta$.

Using the affine parameter $\lambda=2\left(1-a^{5 / 2}\right) / 5$ as the independent variable, equations (26) and (27) for the propagation of $\mathbf{D}$ can be written as

$\frac{d^{2} \delta \mathrm{D}_{1}}{d \lambda^{2}}-\mathscr{R} \delta \mathrm{D}_{1}=\delta \mathscr{R} \mathrm{D}_{1}, \quad \mathrm{D}_{1}(0)=0, \quad \frac{d \mathrm{D}_{1}}{d \lambda}(0)=1$

$\frac{d^{2} \mathrm{D}_{2}}{d \lambda^{2}}-\mathscr{R} \mathrm{D}_{2}=\mathscr{F} \mathrm{D}_{1}, \quad \mathrm{D}_{2}(0)=\frac{d \mathrm{D}_{2}}{d \lambda}(0)=0$,

where $\delta \mathrm{D}_{1}, \mathrm{D}_{2}, \delta \mathscr{R}$ and $\mathscr{F}$ are regarded as perturbations. $\mathrm{D}_{2}$ is calculated using the unperturbed value of $\mathrm{D}_{1}$. The analysis is simplified if we transform the independent variable to $a=(1-5 \lambda / 2)^{2 / 5}$. The unperturbed values for $D_{1}$ and $\mathscr{R}$ in an EDS universe are

$\mathrm{D}_{1}=2 a\left(1-a^{1 / 2}\right)$

$\mathscr{R}=-(3 / 2) a^{-5}$

and so we have

$\frac{d^{2} \delta \mathrm{D}_{1}}{d a^{2}}-\frac{3}{2 a} \frac{d \delta \mathrm{D}_{1}}{d a}+\frac{3}{2 a^{2}} \delta \mathrm{D}_{1}=2 \delta \mathrm{R}_{0}\left(1-a^{1 / 2}\right)$

$\frac{d^{2} \mathrm{D}_{2}}{d a^{2}}-\frac{3}{2 a} \frac{d \mathrm{D}_{2}}{d a}+\frac{3}{2 a^{2}} \mathrm{D}_{2}=2 \mathrm{~F}_{0}\left(1-a^{1 / 2}\right)$

where we use the relation $\delta \mathscr{R}=a^{-4} \delta \mathscr{R}_{0}, \mathscr{F}=a^{-4} \mathscr{F}_{0}$, valid in linear theory. Note that $\delta \mathrm{D}_{1}$ is purely real. The imaginary part of $\delta \mathrm{D}_{1}$, associated with rotation, only appears at the non-linear level.

It is convenient to take the Fourier transform (denoted with a tilde) of this equation in comoving coordinates, $\boldsymbol{x}$, where

$\boldsymbol{x}=\left(x_{1}, x_{2}, x_{3}\right), \quad x_{1,2}=2\left(1-a^{1 / 2}\right) \xi_{01,2}, \quad x_{3}=2\left(1-a^{1 / 2}\right)$

and let $\hat{x}_{1}, \hat{x}_{2}$ and $\hat{x}_{3}$ be unit basis vectors, and $\hat{k}$ be a unit vector in the direction of $\boldsymbol{k}$. Let us now take $\boldsymbol{x}$ to be very close to the $z$ axis so that $x_{3} \simeq x$. We therefore write

$\delta \mathrm{R}_{0}=\int \frac{d^{3} k}{(2 \pi)^{3}} \delta \tilde{\mathrm{R}}_{0} \mathrm{e}^{i k \cdot x}=-\left(\Phi_{0,11}+\Phi_{0,22}+\Phi_{0,33}\right)=\frac{-3 \delta_{0}}{2}$ 
$F_{0}=\int \frac{d^{3} k}{(2 \pi)^{3}} \tilde{F}_{0} \mathrm{e}^{i k \cdot x}=-\left(\Phi_{0,11}-\Phi_{0,22}+2 i \Phi_{0,12}\right)$

where $\Phi_{0}$ is the comoving (i.e. present) gravitational potential which satisfies Poisson's equation and we have substituted $\rho_{0}=3 / 8 \pi$. This gives

$\tilde{\Phi}_{0}=-3 \tilde{\delta}_{0} / 2 k^{2}, \quad k^{2}=k_{1}^{2}+k_{2}^{2}+k_{3}^{2}$

(where $\tilde{\delta}_{0}$ is the Fourier transform of $\delta_{0}$ ), and

$\mathscr{F}_{0}=-\frac{3 \tilde{\delta}_{0}\left(k_{1}+i k_{2}\right)^{2}}{2 k^{2}}$

which implies that

$\frac{d^{2} \delta \mathrm{D}_{1}}{d a^{2}}-\frac{3}{2 a} \frac{d \delta \mathrm{D}_{1}}{d a}+\frac{3}{2 a^{2}} \delta \mathrm{D}_{1}=-3\left(1-a^{1 / 2}\right) \int \frac{d^{3} k}{(2 \pi)^{3}} \tilde{\delta}_{0} \mathrm{e}^{i k \cdot x}$

$\frac{d^{2} \mathrm{D}_{2}}{d a^{2}}-\frac{3}{2 a} \frac{d \mathrm{D}_{2}}{d a}+\frac{3}{2 a^{2}} \mathrm{D}_{2}=-3\left(1-a^{1 / 2}\right) \int \frac{d^{3} k}{(2 \pi)^{3}} \tilde{\delta}_{0} \frac{\left(k_{1}+i k_{2}\right)^{2}}{k^{2}} \mathrm{e}^{i k \cdot x}$.

These inhomogeneous, second order linear differential equations can be solved subject to boundary conditions (53) to obtain

$\Delta M=\frac{2 \delta \mathrm{D}_{1}}{D_{\mathrm{OS}}}=\frac{3 x^{2}}{2} \int \frac{d^{3} k}{(2 \pi)^{3}} \tilde{\boldsymbol{\delta}}_{0} \frac{j_{1}(\boldsymbol{k} \cdot \boldsymbol{x} / 2)}{\boldsymbol{k} \cdot \boldsymbol{x} / 2} \mathrm{e}^{i \boldsymbol{k} \cdot \boldsymbol{x} / 2}$

and

$p=\frac{2 \mathrm{D}_{2}}{D_{\mathrm{OS}}}=-\frac{3 x^{2}}{2} \int \frac{d^{3} k}{(2 \pi)^{3}} \tilde{\delta}_{0} \frac{\left(k_{1}+i k_{2}\right)^{2}}{k^{2}} \frac{j_{1}(\boldsymbol{k} \cdot \boldsymbol{x} / 2)}{\boldsymbol{k} \cdot \boldsymbol{x} / 2} \mathrm{e}^{i \boldsymbol{k} \cdot \boldsymbol{x} / 2}$

[where $j_{1}(\xi)=\sin (\xi) / \xi^{2}-\cos (\xi) / \xi$ is a spherical Bessel function], valid for $x$ very close to the $z$ axis. In general we have

$p=\frac{2 \mathrm{D}_{2}}{D_{\mathrm{OS}}}=-\frac{3 x^{2}}{2} \int \frac{d^{3} k}{(2 \pi)^{3}} \tilde{\delta}_{0}\left[\left(\hat{x}_{1}+i \hat{x}_{2}\right) \cdot \hat{k}\right]^{2} \frac{j_{1}(\boldsymbol{k} \cdot \boldsymbol{x} / 2)}{\boldsymbol{k} \cdot \boldsymbol{x} / 2} \mathrm{e}^{i \boldsymbol{k} \cdot \boldsymbol{x} / 2}$.

\subsection{Point mass}

It is illuminating to recover the formula for the image polarization produced by a single point mass in an Einstein-de Sitter universe. The magnification fluctuation is zero as the density perturbation along the line-of-sight vanishes. Without loss of generality we set $\boldsymbol{\xi}_{0}=0$ so that $\boldsymbol{k} \cdot \boldsymbol{x}=k_{3} x_{3}$. Let the mass, $M$, be located at $\boldsymbol{x}_{M}$, so that the relative density fluctuation is

$\delta_{0}\left(\boldsymbol{x}^{\prime}\right)=\frac{8 \pi M}{3} \delta\left(\boldsymbol{x}^{\prime}-\boldsymbol{x}_{M}\right)$

(where the Dirac delta function, $\delta(\boldsymbol{x})$, must be distinguished from the relative density fluctuation, $\delta_{0}$ ).

Its Fourier transform is then

$\tilde{\delta}_{0}(\boldsymbol{k})=\frac{8 \pi M}{3} \mathrm{e}^{-i \boldsymbol{k} \cdot x_{M}}$.

Substituting into equation (66), and transforming to cylindrical polar coordinates for $\boldsymbol{k}$ and $\boldsymbol{x}_{M}$ we obtain

$p=-4 \pi M x_{3}^{2} \int_{-\infty}^{\infty} \frac{d k_{3}}{2 \pi} \mathrm{e}^{i \kappa x}\left[\frac{j_{1}(\kappa)}{\kappa}\right] \int_{0}^{\infty} \frac{d k_{\perp}}{2 \pi} \frac{k_{\perp}^{3}}{k^{2}} \int_{0}^{2 \pi} \frac{d \phi}{2 \pi} \mathrm{e}^{i\left(2 \phi-k_{\perp} x_{M} \cos \phi\right)}$,

where $k_{\perp}=\left(k_{1}^{2}+k_{2}^{2}\right)^{1 / 2}, x_{M \perp}=\left(x_{M 1}^{2}+x_{M 2}^{2}\right)^{1 / 2}, \kappa=k_{3} x_{3} / 2$ and $\chi=1-2 x_{M 3} / x_{3}$. Performing the $\phi$ and $k_{\perp}$ integrations we obtain

$|p|=\int_{-\infty}^{\infty} \frac{d \kappa}{2 \pi} \kappa j_{1}(\kappa) K_{2}\left(2 x_{M \perp} \kappa / x_{3}\right) \mathrm{e}^{i \kappa x}$. 
Now, we know that only the gravitational component resolved perpendicular to the line-of-sight is effective in deflecting the rays. Correspondingly, in Fourier space the contributing components have $k_{\|} \ll k_{\perp} \sim x_{M \perp}^{-1}$. Hence the argument of the MacDonald function $K_{2}$ is small and we can write $K_{2}(\xi) \simeq 2 / \xi^{2}$. The final, $\kappa$, integral is now elementary and we obtain

$|p|=\frac{8 M x_{M 3}\left(x_{3}-x_{M 3}\right)}{x_{M \perp}^{2} x_{s}}$

in agreement with equation (37).

This calculation motivates us to make a similar approximation in computing the two point polarization correlation function. The phase of the image polarization $p$ is just twice the position angle of the mass with respect to the line-of-sight. If we average over many lines-of-sight at fixed $x_{M \perp}$, the mean value of $p$ will vanish. However, the mean square value will not vanish.

\subsection{Polarization correlation function}

In general the two-point image polarization correlation function for a pair of galaxies located at $\boldsymbol{x}$ and $\boldsymbol{x}^{\prime}$ is given by

$\left\langle p(\boldsymbol{x}) p^{*}\left(\boldsymbol{x}^{\prime}\right)\right\rangle=\frac{9}{4} x_{3}^{2} x_{3}^{\prime 2} \int \frac{d^{3} k}{(2 \pi)^{3}} \int \frac{d^{3} k^{\prime}}{(2 \pi)^{3}}\left\langle\tilde{\delta} \tilde{\delta}^{\prime} *\right\rangle\left[\left(\hat{x}_{1}+i \hat{x}_{2}\right) \cdot \hat{k}\right]^{2}\left[\left(\hat{x}_{1}^{\prime}-i \hat{x}_{2}^{\prime}\right) \cdot \hat{k}^{\prime}\right]^{2}\left[\frac{j_{1}(\boldsymbol{k} \cdot \boldsymbol{x} / 2)}{\boldsymbol{k} \cdot \boldsymbol{x} / 2}\right]\left[\frac{j_{1}\left(\boldsymbol{k}^{\prime} \cdot \boldsymbol{x}^{\prime} / 2\right)}{\boldsymbol{k}^{\prime} \cdot \boldsymbol{x} \boldsymbol{x}^{\prime} / 2}\right] \exp \left[\frac{i\left(\boldsymbol{k} \cdot \boldsymbol{x}-\boldsymbol{k}^{\prime} \cdot \boldsymbol{x}^{\prime}\right)}{2}\right]$.

We interpret the average, \langle\rangle , as either an average over an ensemble of universes or, granted the apparent isotropy of our universe, an average over many different directions. It is this average that we propose to estimate using observations over a few fields. If the density fluctuations are randomly phased and therefore obey Gaussian statistics, then we can introduce a power spectrum of density fluctuations, $P(k)$, defined using

$\left\langle\tilde{\delta} \tilde{\delta}^{\prime *}\right\rangle=(2 \pi)^{6} P(k) \delta\left(\boldsymbol{k}-\boldsymbol{k}^{\prime}\right)$.

For a discussion of the normalization of the power spectrum for models such as CDM and HDM see Section 6.7. Integrating over the delta function we obtain

$\left\langle p(\boldsymbol{x}) p^{*}\left(\boldsymbol{x}^{\prime}\right)\right\rangle=\frac{9}{4} x_{3}^{2} x_{3}^{\prime 2} \int d^{3} k P(k)\left[\left(\hat{x}_{1}+i \hat{x}_{2}\right) \cdot \hat{k}\right]^{2}\left[\left(\hat{x}_{1}^{\prime}-i \hat{x}_{2}^{\prime}\right) \cdot \hat{k}\right]^{2}\left[\frac{j_{1}(\boldsymbol{k} \cdot \boldsymbol{x} / 2)}{\boldsymbol{k} \cdot \boldsymbol{x} / 2}\right]\left[\frac{j_{1}\left(\boldsymbol{k} \cdot \boldsymbol{x}^{\prime} / 2\right)}{\boldsymbol{k} \cdot \boldsymbol{x} / 2}\right] \mathrm{e}^{i \boldsymbol{k} \cdot\left(\boldsymbol{x}-\boldsymbol{x}^{\prime}\right) / 2}$.

Let us first consider two galaxies at the same redshift, we can then replace $x$ and $x^{\prime}$ in equation (75) by $x$. Let us now choose for simplicity the direction of the unprimed ray to measure the polar direction for spherical polar coordinates for the $k$-space integral. Making the small angle approximation and recalling that the contributing Fourier components are nearly perpendicular to the line-of-sight we have,

$\boldsymbol{x}=x(0,0,1), \quad \boldsymbol{x}^{\prime} \simeq x(\theta, 0,1), \quad \boldsymbol{k} \simeq k(\cos \phi, \sin \phi, \mu)$,

where $\cos ^{-1} \mu$ and $\phi$ are the usual spherical polar angles and $|x|=\left|x^{\prime}\right| \simeq x_{3} \equiv x$ - consistent with the approximation $\left[\left(k_{1}^{2}+k_{2}^{2}\right) / k^{2}\right]^{2} \sim 1$. An immediate consequence of making this approximation is that the magnification correlation function is identical to the polarization correlation function:

$\left\langle M(\boldsymbol{x}) M\left(\boldsymbol{x}^{\prime}\right)\right\rangle=\left\langle p(\boldsymbol{x}) p^{*}\left(\boldsymbol{x}^{\prime}\right)\right\rangle$.

After making these substitutions we obtain

$$
\begin{aligned}
\left\langle p(\boldsymbol{x}) p^{*}\left(\boldsymbol{x}^{\prime}\right)\right\rangle & =C_{\mathrm{pp}}(\theta, x)=C_{M M}(\theta, x) \\
& =\frac{9}{4} x^{4} \int_{0}^{\infty} d k k^{2} P(k) \int_{-\infty}^{\infty} d \mu \int_{0}^{2 \pi} d \phi\left[\frac{j_{1}[k x \mu / 2]}{k x \mu / 2}\right]\left[\frac{j_{1}[(k x / 2)(\mu+\theta \cos \phi)]}{(k x / 2)(\mu+\theta \cos \phi)}\right] \exp \left(\frac{i k x \theta \cos \phi}{2}\right),
\end{aligned}
$$

where we have changed the limits of the $\mu$ integration to $\pm \infty$ consistent with our approximation. It is convenient to invoke the correlation theorem at this stage to transform the $\mu$ integration:

$C_{\mathrm{pp}}(\theta, x)=\frac{9 \pi x^{3}}{16} \int_{0}^{\infty} d k k P(k) \int_{-1}^{1} d q\left(1-q^{2}\right)^{2} \int_{0}^{2 \pi} d \phi \mathrm{e}^{i k x \theta \cos \phi(q+1) / 2}$

where we have used the Fourier transform

$\int_{-\infty}^{\infty} d y \mathrm{e}^{-i q y} \frac{j_{1}(y)}{y}=\frac{\pi}{2}\left(1-q^{2}\right) \Theta(1+q) \Theta(1-q)$

and $\Theta(x)$ is the step function. We change variables to $s=(q+1) / 2$, and obtain

$C_{\mathrm{pp}}(\theta, x)=C_{\mathrm{MM}}(\theta, x)=36 \pi^{2} x^{3} \int_{0}^{\infty} d k k P(k) \int_{0}^{1} d s s^{2}(1-s)^{2} J_{0}(s k x \theta)$. 
The rms image polarization for sources at redshift $z$ is given by $\left[C_{p p}(0, x)\right]^{1 / 2}$ or

$p_{\mathrm{rms}}=4 \pi\left[1-(1+z)^{-1 / 2}\right]^{3 / 2}\left[\frac{3}{5} \int_{0}^{\infty} d k k P(k)\right]^{1 / 2}$

The integral in equation (83) will diverge for power spectra shallower than $P(k) \propto k^{-2}$. For purposes of calculation we use the power spectrum for cold dark matter given by Davis et al. (1985) and the power spectrum for hot dark matter given by Bardeen et al. (1986) (hereafter BBKS). The rms image polarization is plotted as a function of redshift for these two cosmologies in Figs 9 and 10. In Fig. 11 we exhibit the correlation function for angular separations $\theta$ also for these two cosmologies.

Next we calculate the correlation function when the source galaxies are found over a range of redshifts. For simplicity, we assume that the number density of galaxies is constant (per unit comoving volume) up to a maximum redshift. We first compute an expression for the mean polarization $\bar{p}$ along a specific direction. The polarization is an additive quantity so we can do this by integrating equation (66)

$\bar{p}(x)=\frac{3}{x^{3}} \int_{0}^{x} p\left(x^{\prime}\right) x^{\prime 2} d x^{\prime}$.

This can be written as

$\bar{p}(x)=-\frac{3 x^{2}}{2} \int \frac{d^{3} k}{(2 \pi)^{3}} \tilde{\delta}_{0} \frac{\left(k_{1}+i k_{2}\right)^{2}}{k^{2}} f(\boldsymbol{k} \cdot \boldsymbol{x} / 2) \mathrm{e}^{i \boldsymbol{k} \cdot \boldsymbol{x} / 2}$,

where

$f(y)=\frac{3 \mathrm{e}^{-i y}}{y^{5}} \int_{0}^{y} d y^{\prime} y^{\prime 3} j_{1}\left(y^{\prime}\right) \mathrm{e}^{i y^{\prime}}=\frac{1}{2}\left[\frac{j_{1}(y)}{y}+\frac{j_{2}(y)}{y^{2}}-i \frac{j_{2}(y)}{y}\right]$.

The equation for the correlation function for $\bar{p}$ can then be written in the same way as equation (79), then

$\bar{C}_{\mathrm{pp}}(\theta, x)=\left\langle\bar{p}(\boldsymbol{x}) \bar{p}^{*}\left(\boldsymbol{x}^{\prime}\right)\right\rangle$

$$
=\frac{9}{4} x^{4} \int_{0}^{\infty} d k k^{2} P(k) \int_{-\infty}^{\infty} d \mu \int_{0}^{2 \pi} d \phi f\left(\frac{k x \mu}{2}\right) f^{*}\left(\frac{k x}{2}[\mu+\theta \cos \phi]\right) \exp \left(\frac{i k x \theta \cos \phi}{2}\right) .
$$

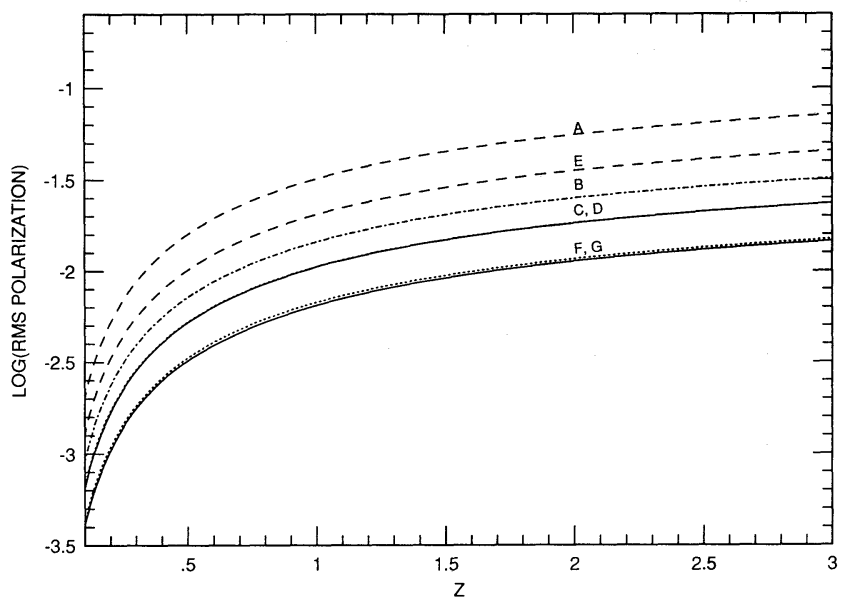

Figure 9. Analytic and $\mathrm{N}$-body results for the rms image polarization versus redshift. Curves A (dashed), B (dot and dash) and $\mathrm{C}$ (dotted) are analytic results (corresponding to smoothing lengths of $0,0.4$ and 0.8 grid cells respectively) and curve D (solid, lying almost on top of curve $\mathrm{C}$ ) is the $\mathrm{N}$-body result for the case that the sources are at one redshift. Curves $\mathrm{E}$ (dashed) and $\mathrm{F}$ (dotted) are analytic results (corresponding to smoothing lengths of 0 and 0.8 grid cells respectively) and curve $\mathrm{G}$ (solid) is the $\mathrm{N}$-body result for the case that the sources are distributed in redshift. Values of $h=1 / 2, \Omega=1$ and $b=2$ were adopted for this figure.

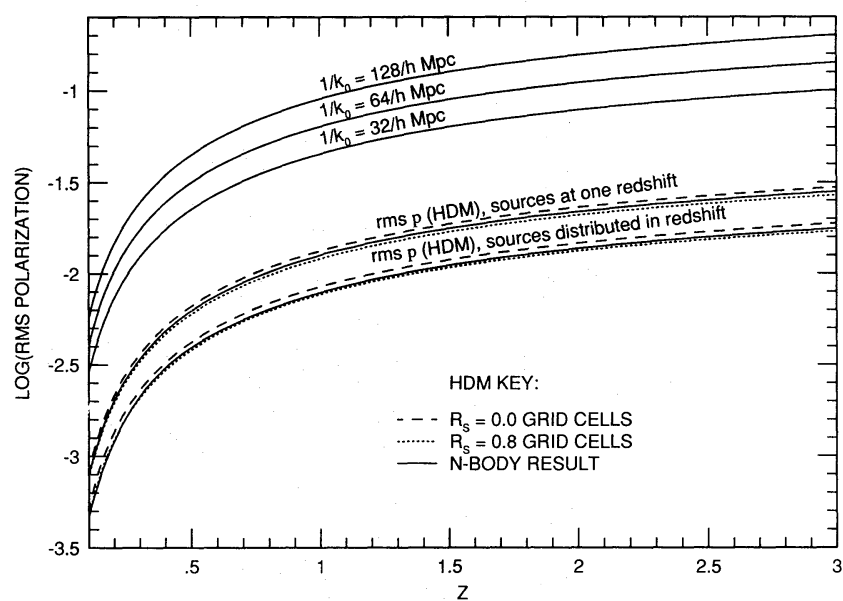

Figure 10. RMS image polarization where the sources are at one redshift for a HDM universe (middle three curves) and one with a delta function power spectrum, $P(k)=A \delta\left(k-k_{0}\right) / k_{0}^{2}, 1 / k_{0}=32$ $h^{-1} \mathrm{Mpc}, 64 h^{-1} \mathrm{Mpc}, 128 h^{-1} \mathrm{Mpc}$ (top three curves) and the rms image polarization where the sources are uniformly distributed in redshift for a HDM universe (bottom three curves). Values of $h=1 / 2, \Omega=1, b=2$ (for HDM) and $b=1$ (for the delta function power spectrum) were adopted for this figure. 

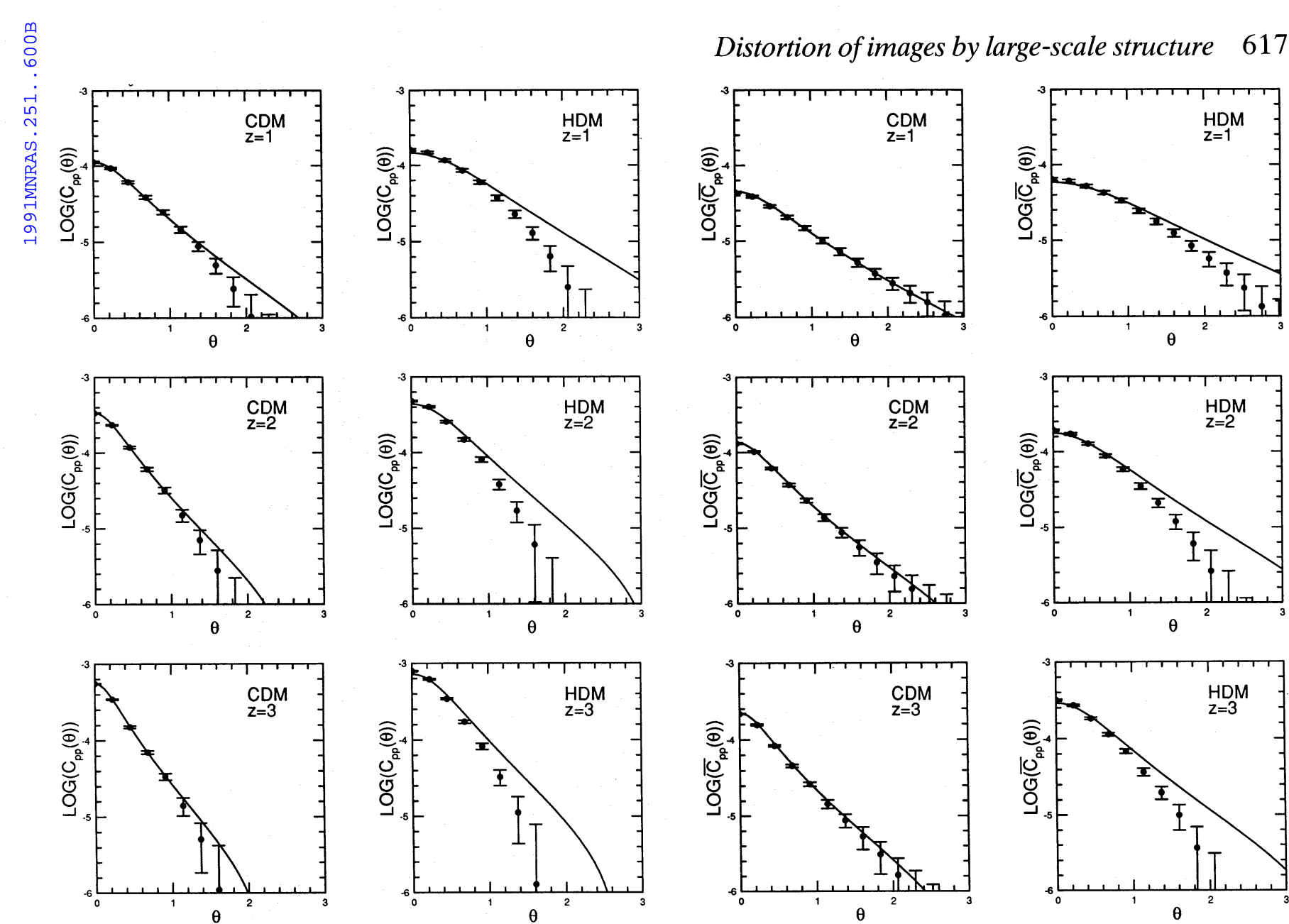

Figure 11. Correlation function for a CDM (left side) and HDM (right side) universes for redshifts of 1,2 and 3 where the sources are at one redshift. Points are $N$-body results; error bars correspond to 1- $\sigma$ scatter between the patches. Lines are analytic results with $r_{\mathrm{s}}=0.8$ grid cells. Values of $h=1 / 2, \Omega=1$ and $b=2$ were adopted for this figure.

Figure 12. Correlation function for a CDM (left side) and HDM (right side) universes for redshifts of 1,2 and 3 where the sources are uniformly distributed in redshift. Points are $\mathrm{N}$-body results; error bars correspond to 1- $\sigma$ scatter between the patches. Lines are analytic results with $r_{\mathrm{s}}=0.8$ grid cells. Values of $h=1 / 2, \Omega=1$ and $b=2$ were adopted for this figure.

Once again, we invoke the correlation theorem to transform the $\mu$ integration using the Fourier transform

$\int_{-\infty}^{\infty} d y \mathrm{e}^{-i q y} f(y)=\frac{\pi}{16}(1-q)\left(1-q^{2}\right)(5+q) \Theta(1+q) \Theta(1-q)$

to obtain

$$
\begin{aligned}
\bar{C}_{\mathrm{pp}}(\theta, x) & =\bar{C}_{\mathrm{MM}}(\theta, x) \\
& =\frac{9 \pi x^{3}}{1024} \int_{0}^{\infty} d k k P(k) \int_{-1}^{1} d q(1-q)^{2}\left(1-q^{2}\right)^{2}(5+q)^{2} \int_{0}^{2 \pi} d \phi \mathrm{e}^{i k x \theta \cos \phi(q+1) / 2} \\
& =36 \pi^{2} x^{3} \int_{0}^{\infty} d k k P(k) \int_{0}^{1} d s s^{2}(1-s)^{4}(1+s / 2)^{2} J_{0}(s k x \theta) .
\end{aligned}
$$

The rms image polarization is given by a similar expression to equation (83)

$\bar{p}_{\mathrm{rms}}=4 \pi\left[1-(1+z)^{-1 / 2}\right]^{3 / 2}\left[\frac{17}{70} \int_{0}^{\infty} d k k P(k)\right]^{1 / 2}$,

which is approximately a factor of 0.64 times the polarization obtained if the sources were all at the maximum redshift.

The angular correlation function $\bar{C}_{\mathrm{pp}}(\theta, x)$ is exhibited in Fig. 12 for CDM and HDM universes. 


\section{$6.4 N$-body simulations}

$N$-body simulations, using Fourier methods, of the growth of fluctuations in Friedman universes have proved to be powerful tools for following the evolution of structure (e.g. White et al. 1987). The Fourier initial conditions are specified by the power spectrum $P(k)$ of density fluctuations plus their statistics. The initial density field is usually taken to be Gaussian which in Fourier space means that the phases of the waves are uncorrelated and random and that the amplitudes of the real and imaginary parts for each mode have Gaussian distribution with zero mean and a variance that equals the power spectrum density $P(k)$. Individual realizations of expanding universes with these density fluctuations are then evaluated dynamically.

We have performed a set of $N$-body simulations in a box that expands with the universe. Density was assigned to the grid using a tri-linear interpolation (CIC) scheme which assigns the mass from a particle to the eight nearest grid points. The gravitational field was solved on the cubic grid with periodic boundary conditions using Fast Fourier Transform (FFT) methods. The force field was calculated at the particle positions using CIC interpolation and at the grid points by two-point differencing of the potential. The particles were advanced using a time-centred leapfrog method with the expansion factor $a$ as time variable.

The initial conditions were generated from a random realization of the Gaussian density field from which the force field was computed. The Zel'dovich approximation was then used to translate the force into displacements and velocities for the particles which started out on a uniform grid. For further detail, see Villumsen (1989) (cf. also Efstathiou et al. 1985).

The calculations were performed in comoving coordinates $x$ expanding with a box of side $L$. Periodic boundary conditions imply zero power on scales larger than $L$. If we have an $N^{3}$ grid and $N_{\mathrm{p}}^{3}$ particles we can generate the wave numbers up to

$\frac{2 \pi}{L} \frac{\min \left(N_{\mathrm{p}}, N\right)}{2}$

in each dimension in the initial conditions, typically $N_{\mathrm{p}} \leq N$. As the system clusters we can specify structures down to about one grid cell depending on the details of the Green's function and the differencing and interpolation schemes, this corresponds at best to a maximum wave number of $(2 \pi / L)(N / 2)$. In these simulations we have $N=N_{\mathrm{p}}=128$ so only 64 waves can be specified in each dimension. In the CDM and HDM simulations, the box size is taken to be $L=500 h^{-1} \mathrm{Mpc}$, where $h=H_{0} / 100 \mathrm{~km} \mathrm{~s}^{-1}$ $\mathrm{Mpc}^{-1}$. We used $h=1 / 2$ which is the preferred value for CDM universes with a linear bias model (e.g. Davis et al. 1985; White et al. 1987). At the present epoch, the rms density fluctuation (with a top hat filter) is

$\left(\frac{\delta \rho}{\rho}\right)_{\mathrm{rms}}\left(8 h^{-1} \mathrm{Mpc}\right)=\sigma_{8}=\frac{1}{b}$,

where $b$ is the linear bias factor (BBKS). We have chosen $b=2$ but the results can be rescaled to other bias factors.

Evolution equations (56) and (57) for $\delta D_{1}$ and $D_{2}$ were integrated backwards from the present along unperturbed rays in comoving coordinates using the fourth-order Runge-Kutta scheme. We assume that the universe is periodic on the scale of the grid and wrap the ray around (avoiding symmetry directions). If we run a light ray along a symmetry direction (i.e. one of the axes of the grid), it will traverse correlated patches of density on the scale of the grid and thus add artificial power on the scale of the grid. If we use a power spectrum with significant power on the scale of the grid or larger, then the assumption of periodicity will induce significant errors, even with avoidance of symmetry directions. Commonly used model universes such as HDM or CDM models based on inflation have very little power on these scales so the assumption of periodicity should not induce significant errors.

For each ray, we find $\boldsymbol{x}(a)$ at each time-step. At each corner of the grid cell in which the ray is located we evaluate the second derivatives of $\Phi$, the potential in comoving coordinates. If the index of a corner of the gridcell is $\boldsymbol{n}=\left(n_{1}, n_{2}, n_{3}\right)$ then

$\Phi_{, 11}=N^{2}\left[\Phi\left(n_{1}-1, n_{2}, n_{3}\right)+\Phi\left(n_{1}+1, n_{2}, n_{3}\right)-2 \Phi\left(n_{1}, n_{2}, n_{3}\right)\right]$

$\Phi_{.12}=\frac{N^{2}}{4}\left[\Phi\left(n_{1}-1, n_{2}-1, n_{3}\right)+\Phi\left(n_{1}+1, n_{2}+1, n_{3}\right)-\Phi\left(n_{1}-1, n_{2}+1, n_{3}\right)-\Phi\left(n_{1}+1, n_{2}-1, n_{3}\right)\right]$

and equivalently. Also $\Phi_{, i j}=\Phi_{, j}$. To calculate the derivatives at the position of the light ray we use CIC interpolation with the values at the eight corners. These derivatives use the coordinate system of the $N$-body code, however, we need the derivatives $\Phi_{, 11}^{\prime} ; \Phi_{, 22}^{\prime} ; \Phi_{, 33}^{\prime}$, and $\Phi_{, 12}^{\prime}$ in a coordinate system where $e_{3}^{\prime}$ is along the ray. There is a rotational degree of freedom in the choice of coordinate system so we choose the following right-handed system:

$\boldsymbol{x}_{3}^{\prime}=(\sin \theta \cos \phi, \sin \theta \sin \phi, \cos \theta) \quad \boldsymbol{x}_{2}^{\prime}=(-\sin \phi, \cos \phi, 0) \quad \boldsymbol{x}_{1}^{\prime}=(\cos \theta \cos \phi, \cos \theta \sin \phi,-\sin \theta)$.

The derivatives are computed in the primed coordinate system using

$\Phi_{. i j}^{\prime}=\sum_{k . l} \Phi_{. k l} \frac{\partial x_{k}}{\partial x_{i}^{\prime}} \frac{\partial x_{l}}{\partial x_{j}^{\prime}}$

The computed magnification and image polarization for typical realizations of CDM and HDM universes are exhibited in Fig. 13. The numerical simulations also verify that the image rotation given by $D_{1 i}$ is completely negligible. The rms value of $D_{1 i} / D_{1 r}$ is only $\sim 6 \times 10^{-5}$ at $z=1$. 

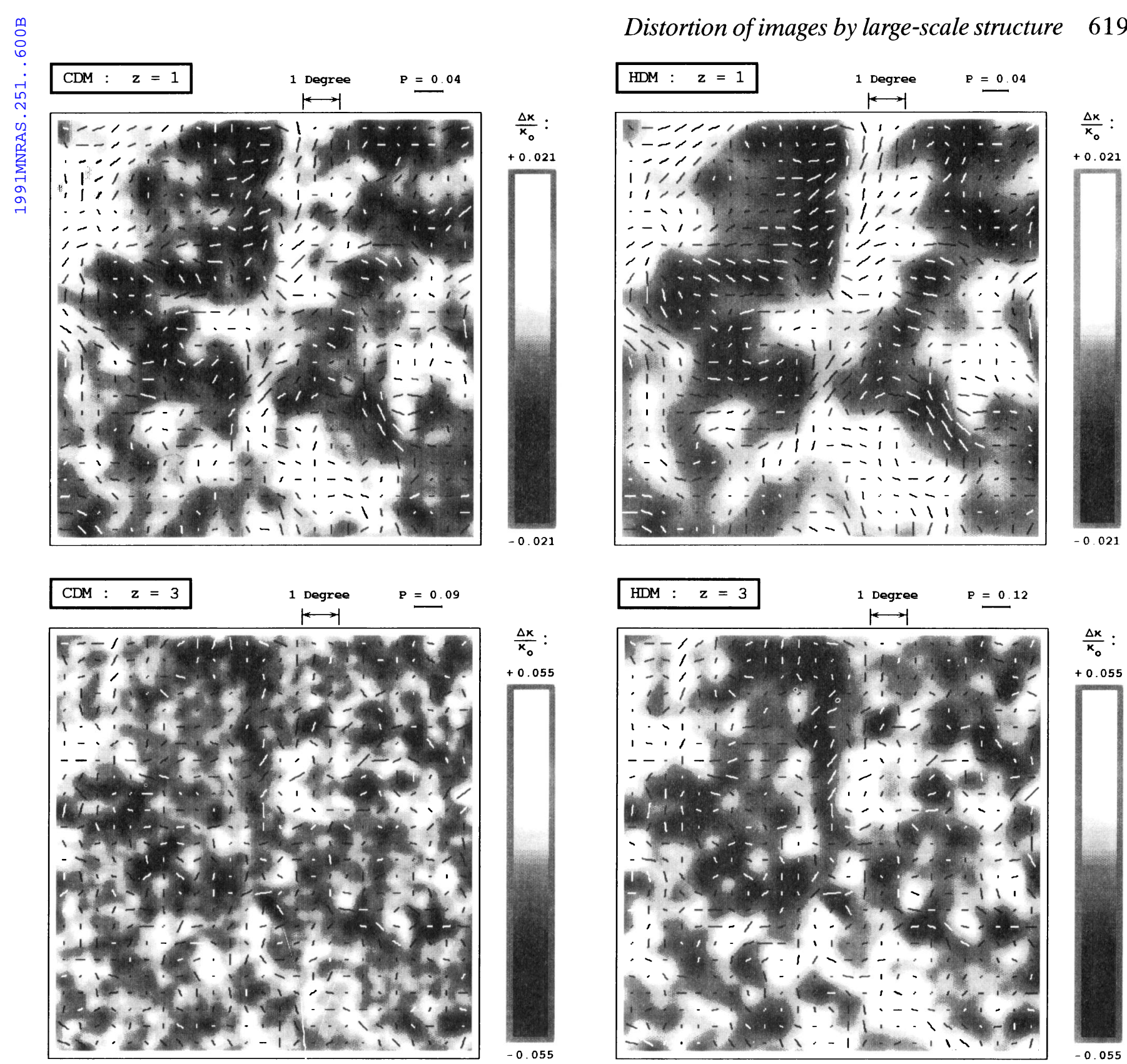

Figure 13. Simulated map of the magnification contours and the polarization $p$ for CDM and HDM universes and source galaxies at redshifts $z=1$ and 3 .

\subsection{Numerical evaluation of the polarization correlation function}

Sixteen observer positions, $\boldsymbol{x}_{0}$, were chosen randomly and the correlation of the polarization on scales up to a few degrees determined. Each observer looked in a random direction at $50 \times 50$ light rays in a square patch $0.2 \mathrm{rad}=11.5^{\circ}$ across so our resolution is $0.23^{\circ}$.

We computed $\mathrm{D}_{2}$ for each of the $N \times N(N=50)$ light rays (for given redshifts) in each of $M=16$ patches. The correlation function was computed independently in each of the patches:

$\mathrm{C}_{\mathrm{pp}}^{m}(\theta)=\frac{4\left\langle\mathrm{D}_{2}(\boldsymbol{x}) \mathrm{D}_{2}^{*}\left(\boldsymbol{x}^{\prime}\right)\right\rangle}{\mathrm{D}_{\mathrm{Ir}}^{2}}, \quad m=1,2, \ldots, M ; \quad \cos (\boldsymbol{\theta})=\frac{\boldsymbol{x} \cdot \boldsymbol{x}^{\prime}}{|\boldsymbol{x}|\left|\boldsymbol{x}^{\prime}\right|}$,

where $\theta$ is the angular distance between the rays $i$ and $j$ and we average over all pairs of rays separated by an angle lying in a bin centred on $\theta$. The correlation function for a particular redshift was determined by taking the average of $C_{\mathrm{pp}}^{m}(\theta)$ over the patches and subtracting a correction term,

$C_{\mathrm{pp}}(\theta)=\frac{1}{M} \sum_{m=1}^{M} \mathrm{C}_{\mathrm{pp}}^{m}(\theta)-\frac{4\left\langle\mathrm{D}_{2}\right\rangle^{2}}{\mathrm{D}_{1 \mathrm{r}}^{2}}$ 
where the average, \langle\rangle is taken over all the patches. The mean of $C_{p p}(\theta)$ over the entire sky should be zero. However, in our individual finite-sized patches the mean of $C_{\mathrm{pp}}(\theta)$ is not exactly zero and, so, the 'true' correlation function is not merely the mean of the correlation functions from each of the individual patches. We make a first order correction to our calculation of $C_{\mathrm{pp}}(\theta)$ by subtracting off the second term in equation (98). However, this correction makes very little difference to the observed correlation function.

The variance in the correlation function is

$\sigma(\theta)^{2}=\frac{1}{M-1} \sum_{m=1}^{M}\left[\mathrm{C}_{\mathrm{pp}}^{m}(\theta)-C_{\mathrm{pp}}(\boldsymbol{\theta})\right]^{2}$.

\subsection{Smoothing the power spectrum}

In order to compare the analytical and $N$-body results directly, we must take into account the smoothing of the force field that is encountered in the $N$-body simulation. To this end, both the finite resolution of the code ( 1 grid cell) and the fact that the potential is differenced (as opposed to differentiated) in order to determine the forces must be considered. The combination of these two factors amounts to a smoothing length of approximately 2 grid cells (see Villumsen 1989, fig. 1). We modify the power spectrum, $\mathrm{P}(k)$, by multiplying it by a Gaussian smoothing function

$P(k)=\mathrm{e}^{-k^{2} r_{s}^{2}} \mathrm{P}(k)$,

where $\mathrm{P}(k)$ is the unfiltered power spectrum and the smoothing length is $r_{\mathrm{s}}$. We choose $r_{\mathrm{s}}$ so that the Gaussian smoothing volume and the $N$-body smoothing volume are the same, $(2 \pi)^{3 / 2} r_{\mathrm{s}}^{3}=2^{3}$, leaving us with $r_{\mathrm{s}}=0.8$ grid cells (recall that one grid cell is $3.8 h^{-1} \mathrm{Mpc}$ so the smoothing length is $3.1 h^{-1} \mathrm{Mpc}$ ). We expect that if non-linear effects are not important the analytic results with a smoothing length of 0.8 grid cells should agree well with the $N$-body results.

We have introduced the smoothing of the power spectrum primarily to relate the analytical results to the $N$-body calculations. However, there is a more important reason for smoothing the density fluctuation spectrum and this concerns the manner in which the polarization will have to be measured observationally. In order to obtain a sufficiently accurate value for the polarization at a point, it will be necessary to average over a finite area of sky. This procedure will effectively erase any evidence for smaller scale structure. The manner in which this smoothing is carried out is dependent upon the details of the image polarization survey. However, once this is settled, precise predictions for the smoothed correlation function can be computed for a given power spectrum of density fluctuations and world model. Some specific examples will be presented in a future paper.

\subsection{Normalizing the power spectrum}

In order to compute the analytical image polarization and correlation functions one must know the normalization of the power spectrum. The power spectrum may be written as $\mathrm{P}(k)=A k T^{2}(k)$, where $T(k)$ is the transfer function and $A$ is a normalization constant. The mean-square mass fluctuation inside a sphere of radius $R$ is

$\left[\frac{\Delta M}{M}(R)\right]^{2}=\int d^{3} k \mathrm{P}(k) W^{2}(k R)=\int d^{3} k A k T^{2}(k) W^{2}(k R)$,

where

$$
W(k R)=\frac{3}{(k R)^{3}}[\sin (k R)-k R \cos (k R)]=\frac{3 j_{1}(k R)}{k R}
$$

(e.g. Peebles 1980). For $R=8 h^{-1} \mathrm{Mpc}$ we introduce the definition of the linear bias factor, $b,(\mathrm{BBKS})$ and therefore the normalization constant may be computed as

$A=\left[b^{2} \int d^{3} k k T(k) W^{2}\left(8 h^{-1} \mathrm{Mpc} k\right)\right]^{-1}$.

For $h=0.5$ we find $A_{\mathrm{CDM}}=1.78 \times 10^{4} b^{-2} \mathrm{Mpc}^{-2}$ and $A_{\mathrm{HDM}}=5.78 \times 10^{4} \mathrm{~b}^{-2} \mathrm{Mpc}^{-2}$. The normalization of the power spectra was computed without using the Gaussian filter in the calculation of the image polarization correlation function because the $N$-body simulation attempts to reproduce the correct unsmoothed power spectrum at large scales.

\subsection{Non-linear evolution}

Full $N$-body simulations in which the density fluctuations were allowed to grow to non-linear amplitude were performed for CDM and HDM models. The rms image polarization, $p_{\mathrm{rms}}(x)$, and the correlation function $C_{\mathrm{pp}}(\theta)$, (as a function of $\theta$ for various redshifts) were then evaluated. Initial conditions starting at a redshift $z_{\mathrm{i}}=16$ (so that $\sigma_{8}=1 / 34$ ) were generated and the simulation was evolved forward in time for 17 expansion factors with a steplength $\Delta a=1 / 340$. (Linearized $N$-body calculations were also performed and the results differ from the full $N$-body simulations by a few per cent.) 
The rms image polarization as a function of redshift for a CDM universe for the case where the sources are at one redshift (equation 83) is shown in Fig. 9. The $N$-body result (curve D) and analytical results for Gaussian smoothing lengths of zero (curve A) and 0.8 grid cells (curve $\mathrm{C}$, which lies almost directly on top of curve D) are shown. For comparison the analytical image polarization for a Gaussian smoothing length of 0.4 grid cells, corresponding to a smoothing length of one grid cell in the code, is also shown. All four curves have essentially the same functional form, their shapes agreeing well over the entire range of $z$, however, their amplitudes vary with the choice of smoothing length used in the analytical calculation or the use of a grid (in the case of the $\mathrm{N}$-body simulations). The amplitude of the analytical curve for a smoothing length of zero is approximately three times that of the $N$-body curve, while the amplitude of the analytical curve for a smoothing length corresponding to 0.8 grid cells (which is what is expected to occur in the $N$-body code) is, to two significant digits, the same as that of the $N$-body curve. Thus, we observe that the functional form of the rms image polarization function is nearly independent of the smoothing length, but the amplitude depends sensitively on the smoothing length used. (See Table 1 for a summary of these results for redshifts of 1, 2 and 3.) A question that should be addressed is the size of a simulation we would require to make the $N$-body curve approach the analytical, unsmoothed curve. If we increase the size of the simulation to $1024^{3}$ grid cells (i.e. we decrease our smoothing length by a factor of 8 ) we increase the amplitude of $D_{2}$ by a factor of 2 . Since the amplitude of the analytical curve is approximately three times the amplitude of the $\mathrm{N}$-body curve, we see there is little to be gained by increasing the number of grid cells.

We find that our results differ significantly from those published by Jaroszyński et al. (1990). These authors calculated the probability distribution of the ellipticity induced in individual, circular galaxy images obtaining a mean value of $\sim 0.043$ at $z=1$, significantly larger than our computation (and analytical calculation) of $C_{\mathrm{pp}}(0)^{1 / 2}=0.011$. There are two factors which, in combination, may explain the discrepancy between the two sets of results. First, the grid used by Jaroszyński et al. is $128 h^{-1}$ $\mathrm{Mpc}$ on a side (compared to ours which is $500 \mathrm{~h}^{-1} \mathrm{Mpc}$ ) and both grids have $128^{3}$ grid cells. This means that if the smoothing length for Jaroszyński et al's calculation is taken to be 2 grid cells (as we took ours to be), their smoothing length is equivalent to 0.256 of our grid cells (corresponding to a Gaussian smoothing length, $r_{\mathrm{s}}$, of $0.8 h^{-1} \mathrm{Mpc}$ ). According to our analytic calculations of the rms image polarization, at a redshift of one the amplitude of the function for a smoothing length of $0.8 h^{-1}$ Mpc should be approximately 1.7 times the amplitude of the function for a smoothing length of $3.1 h^{-1} \mathrm{Mpc}$, which is the Gaussian smoothing length we have assumed. Also, our $N$-body calculations differ from those of Jaroszyński et al. in that we specifically avoided running light rays along symmetry directions while they specifically ran all the rays along symmetry directions. We ran another simulation in which all the light rays ran along symmetry directions and found that for a redshift of one the rms image polarization was approximately a factor of 1.8 higher than the simulation in which symmetry directions were specifically avoided. The combination of these two factors leads us to expect the results of Jaroszyński et al. to be a factor of $1.7 \times 1.8 \simeq 3.1$ higher than our numerical results.

The rms image polarization as a function of redshift for a HDM universe for the case where the sources are at one redshift (equation 83) is shown in Fig. 10. Both analytical curves differ from the $N$-body results by approximately 5 per cent. In contrast to the case of CDM, we observe that both the shapes and amplitudes of the analytic and $N$-body image polarization functions agree well over the entire range of $z$, which is due to the sharp cut-off of the HDM power spectrum. That is, since the power spectrum is cut off sharply, filtering it on scales smaller than the cut-off frequency will have very little effect.

Also shown in Figs 9 and 10 is the rms image polarization for the case where the sources are uniformly distributed in redshift (equation 91). Analytic results for Gaussian smoothing lengths of 0,0.4 (CDM only) and 0.8 grid cells and the $N$-body results are shown. Again we note that the functional form of the averaged image polarization function is nearly independent of smoothing length but the amplitude is not. (See Table 2 for a summary of these results for redshifts of 1,2 and 3 .)

The polarization correlation functions were also calculated for the $N$-body simulations up to an angular separation of $3^{\circ}$. These are superposed upon the analytical results in Figs 11 (single source redshift) and 12 (distributed sources). The error bars are the 1- $\sigma$ uncertainty in the mean derived from the scatter between the 16 patches. In both cases the agreement is good for small $\theta$ and, in the case of single source redshift, the agreement is better at large redshift, whereas in the case of distributed sources the agreement is better at small redshift. The analytical correlation function crosses zero later than the $N$-body estimate in all six cases shown. Overall, the agreement is better in the case of CDM than HDM.

The accuracy of the paraxial approximation increases the greater the number of wavelengths between the observer and the source. In the case of HDM essentially all the power comes from waves with $1 / k_{0} \sim 20 h^{-1} \mathrm{Mpc}$, of which there are only 70 wavelengths out to $z=1$. In CDM there is a significant amount of power on smaller scales so there are more wavelengths out to the source. Thus we expect the agreement between the $N$-body and analytic results to be better for CDM than for HDM. Another source of discrepancy comes from the finite number of waves in the simulation, which introduces shot noise. Only the small fraction of waves that are nearly perpendicular to the line-of-sight contribute significantly to the shear. Shot-noise effects can

Table 1. Analytic and numerical results for the rms image polarization measured in per cent for CDM and HDM universes in which the sources are at one redshift and the smoothing length $r_{\mathrm{s}}$ is varied.

$z$

$\begin{array}{cccc} & r_{s}=0.0 & r_{s}=0.8 & \text { N-body } \\ 1 & 3.3 & 1.1 & 1.1 \\ 2 & 5.6 & 1.9 & 1.9 \\ 3 & 7.2 & 2.4 & 2.4\end{array}$

$\mathrm{CDM}$

2.4
HDM

$\begin{array}{cc}r_{s}=0.8 & \text { N-body } \\ 1.2 & 1.3 \\ 2.1 & 2.2 \\ 2.7 & 2.8\end{array}$


thus be important in these simulations, especially for the HDM simulations. This shows up most strongly for HDM in $C_{\mathrm{pp}}(\theta)$ at large angles where there is a significant discrepancy, while for CDM the agreement is excellent.

Fig. 14 shows how $C_{\mathrm{pp}}(\theta)$ is affected by smoothing the power spectrum. The plots on the left side of the figure show the analytical predictions for $C_{\mathrm{pp}}(\theta)$ for $z=1,2$ and 3 for smoothing lengths of 0 and 0.8 grid cells for CDM. The plots on the right side of the figure show the corresponding results for HDM. The angles at which the curves fall to half their peak value are indicated by circles. We note that smoothing has little effect on the HDM curves and a significant effect on the CDM curves. Again, this is due to the shapes of the respective power spectra.

The correlation length is often taken to be the point at which the correlation function crosses zero, $\theta_{0}$. In principle this is an attractive definition, but in practice it is a difficult number to measure accurately (since the functions become very flat near zero) and is sensitive to smoothing. One could take the point at which the correlation function reaches half its maximum to be the correlation length, however, this number is very sensitive to smoothing (see Fig. 14). (In the case of a hot dark matter dominated universe this would not be a problem, since we have seen that smoothing the HDM power spectrum has little effect on the correlation function, however, since we do not know that the universe is hot dark matter dominated this is not a particularly attractive choice.) An alternative definition of the correlation length is $\theta_{1 / 2}$ as defined by equation (52) which is easier to measure than the first definition and is less sensitive to smoothing than the second definition.

The integrated correlation function assuming a smoothing length of 0.8 grid cells is shown in Fig. 15. The analytic values of $\theta_{1 / 2}$ for redshifts of 1, 2 and 3 are shown in Table 3 for CDM and HDM models for smoothing lengths of 0 and 0.8 grid cells. The correlation lengths and amplitudes of the correlation functions are similar for both the CDM and HDM models, however, the shape of the functions are quite different. For the HDM model the correlation function decreases slowly with angle while for the CDM model it falls off more quickly. Thus, the integral of the correlation function out to $\theta_{0}$ is larger for the HDM than for CDM. We also note that the correlation length is inversely proportional to $x$.

\subsection{Delta function power spectrum}

Recent observations (e.g. Lynden-Bell et al. 1988; Broadhurst et al. 1990; Maddox et al. 1990) indicate that there may be significant power on scales larger than $\sim 20 h^{-1} \mathrm{Mpc}$ and possibly even a spike at $128 h^{-1} \mathrm{Mpc}$, which motivates us to investigate the effect on the correlation function of putting power on large scales. As an illustrative 'toy' model we choose a delta function power spectrum,

$P(k)=A \delta\left(k-k_{0}\right) / k_{0}^{2}$,
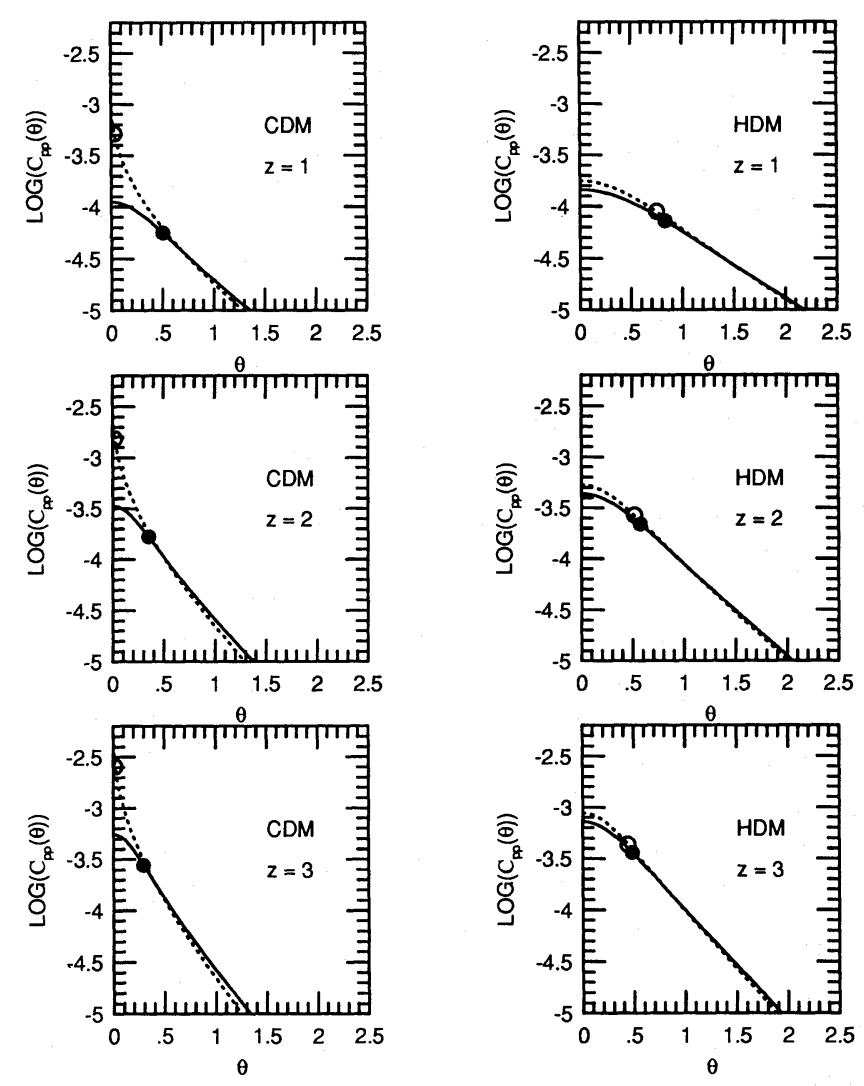

Figure 14. Analytical results for $C_{\mathrm{pp}}(\theta)$ for sources at one redshift for $\mathrm{CDM}$ (left side) and HDM (right side) universes for smoothing lengths, $r_{\mathrm{s}}$, of 0 (dotted line) and 0.8 (solid line) grid cells. The point at which $C_{\mathrm{pp}}(\theta)=0.5 C_{\mathrm{pp}}(0)$ is indicated by circles (open circles for $r_{\mathrm{s}}=0$ grid cells; filled circles for $r_{\mathrm{s}}=0.8$ grid cells). Values of $h=1 / 2, \Omega=1$ and $b=2$ were adopted for this calculation. 
Table 2. Analytic and numerical results for the rms image polarization measured in per cent for CDM and HDM universes in which the sources are uniformly distributed in redshift and the smoothing length $r_{\mathrm{s}}$ is varied.

$\begin{array}{ccccccc}z_{\max } & & \text { CDM } & & & \text { HDM } & \\ & r_{s}=0.0 & r_{s}=0.8 & \text { N-body } & r_{s}=0.0 & r_{s}=0.8 & \text { N-body } \\ 1 & 2.1 & 0.69 & 0.66 & 0.87 & 0.78 & 0.80 \\ 2 & 3.6 & 1.2 & 1.1 & 1.5 & 1.3 & 1.4 \\ 3 & 4.6 & 1.5 & 1.5 & 1.9 & 1.7 & 1.8\end{array}$

Table 3. Analytic results for the correlation length, $\theta_{1 / 2}$, for CDM and HDM universes with different smoothing lengths.

$z \quad$ CDM

$\begin{array}{ccccc} & r_{s}=0.0 & r_{s}=0.8 & r_{s}=0.0 & r_{s}=0.8 \\ 1 & 0.7 & 0.9 & 1.1 & 1.2 \\ 2 & 0.5 & 0.6 & 0.8 & 0.8 \\ 3 & 0.4 & 0.5 & 0.6 & 0.7\end{array}$
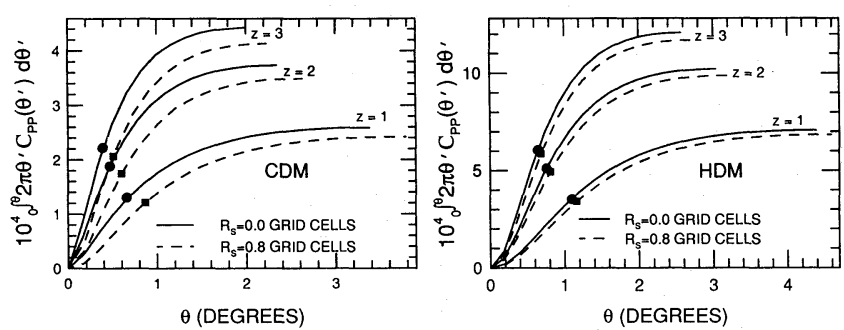

Figure 15. Analytical results for the integrated cross correlation function where the sources are at one redshift for CDM (left panel) and HDM (right panel) universes. Smoothing lengths of 0 (solid line) and 0.8 (dashed line) grid cells were used. $\theta_{1 / 2}$ is indicated by filled circles for $r_{\mathrm{s}}=0$ grid cells and by filled squares for $r_{\mathrm{s}}=0.8$ grid cells. Values of $h=1 / 2, \Omega=1$ and $b=2$ were adopted for this calculation.
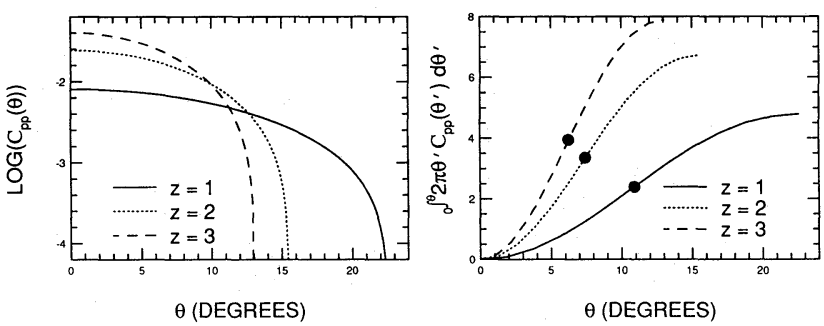

Figure 16. The left panel shows the analytical correlation function for a universe with a delta function power spectrum, $P(k)=A \delta\left(k-k_{0}\right) / k_{0}^{2}, 1 / k_{0}=128 h^{-1} \mathrm{Mpc}$, for source redshifts of 1,2 and 3 . The right panel shows the analytic result for the integrated cross correlation function for redshifts of 1,2 and 3 where all the sources are at one redshift. Filled circles denote the correlation length $\theta_{1 / 2}$. Values of $h=1 / 2, \Omega=1$ and $b=1$ were adopted for this figure.

where $k_{0}$ corresponds to a scale $L$, the scale on which there is extra power. This calculation is only meant to show that largescale power gives a significant polarization signal. If we assume the small angle approximation holds then from equations (82) and (101) we see that the correlation function for the delta function power spectrum in terms of $\delta \rho / \rho$ on a given physical scale $x_{0}$ is

$C_{\mathrm{pp}}(\theta)=\frac{9 \pi x^{3}}{k_{0}}\left[\frac{\delta \rho}{\rho}\left(x_{0}\right)\right]^{2} \int_{0}^{1} d s s^{2}(1-s)^{2} J_{0}\left(s k_{0} x \theta\right)$

which is valid provided $k_{0} x_{0}<1$. (Note that $x_{0}$ above has physical units whereas $x$ is a dimensionless number.) By setting $\theta=0$ in the above equations we see that the image-polarization scales as the square root of $L$. The 's' integral has $k_{0} x \theta$ as a factor in the argument for $J_{0}$ so the correlation length is proportional to $L$ and inversely proportional to $x$.

The left-hand panel of Fig. 16 shows $C_{\mathrm{pp}}(\theta)$ for a delta function power spectrum with $1 / k_{0}=128 \mathrm{~h}^{-1} \mathrm{Mpc}$ and the right-hand panel shows the integrated correlation function out to $\theta_{0}$. The functions in this figure were calculated using the full $3 \mathrm{D}$ integral for $C_{\mathrm{pp}}(\theta)$, rather than equation (105), since the full 3D integral is valid at the angular scales in the problem, which approach $25^{\circ}$. In practice, however, the results given by equation (105) vary little from those given by the full 3D integral, the agreement being best for smaller angles, or course. We find that the rms image polarization is $\sim 0.1(\delta \rho / \rho)$ and the correlation length, $\theta_{1 / 2}$, is $\sim$ $11^{\circ}$ for sources at a redshift $z=1$.

\subsection{Scaling relations}

Simple scaling relations for the shear can be derived from equations (82) and (90). We see that $C_{\mathrm{pp}}(\theta, x)=x^{3} g(x \theta)$, where $g$ is a function of the power spectrum and the number density of sources a function of $x$. Thus $C_{\mathrm{pp}}(\theta, x)=x^{3} C_{\mathrm{pp}}(x \theta, 1)$. With this 


\section{R. D. Blandford et al.}

scaling, $\theta_{1 / 2}$ and $\theta_{0}$ are inversely proportional to $x$. Furthermore, the integrated correlation function is proportional to $x$. These scaling relations are valid for any source distribution in $x$. For the delta function power spectra we see that the integrated correlation function scales as $L^{3}$.

\section{DISCUSSION}

In the present paper, we have shown how large-scale structure can induce a surprisingly large distortion of the images of distant galaxies that can be correlated over angular scales $\sim 0.5-1^{\circ}$. We found that standard cold and hot dark matter cosmogonies or purely kinematical descriptions in which roughly 10 per cent of the closure density is distributed in voids and walls with characteristic scale size $\sim 100 \mathrm{~h}^{-1} \mathrm{Mpc}$ produce a correlated ellipticity of magnitude a few per cent, comparable with the limit of Valdes et al. (1983). Larger ellipticities are predicted if the observed galaxies are less biased tracers of the mass in the universe. Observationally it will be difficult to distinguish between CDM and HDM universes. However, since the larger the scale the power is put on, the larger signal one expects to observe, it should be relatively easy to disprove observationally models with large scales in the mass distribution.

We therefore propose that maps of image polarization might provide a powerful cosmological diagnostic of the distribution of mass on scales $\sim 100 \mathrm{Mpc}$. We would, in effect, be using photons in place of galaxies as dynamical tracers of the gravitational field (at least adopting the Newtonian approximation to general relativity).

This proposal is made more viable by the discovery of a surprisingly large population of apparently high-redshift galaxies (Tyson 1988; Tyson \& Seitzer 1988). These galaxies are reported to have a sky density of $\sim 3 \times 10^{5} \mathrm{deg}^{-2}$ at $B=28 \mathrm{mag}$ roughly four times greater than the density of comoving $L^{*}$ galaxies out to a redshift $z=3$. Tyson and collaborators (e.g. Guhathakurta, Tyson \& Majewski 1991) have argued that these galaxies have angular sizes $\sim 2-3$ arcsec, are very blue and mostly distributed between redshifts $1<z<3$. More recently, though, Lilly, Cowie \& Gardner (1991), while measuring a comparable $\left(2.5 \times 10^{5}\right.$ $\left.\mathrm{deg}^{-2}\right)$ sky density, find that the galaxies are $\sim 0.8$ mag redder and significantly smaller $(\sim 0.5-0.8$ arcsec $)$ than found by Tyson and collaborators. Roughly half the light from these galaxies comes from only 1 per cent of the sky. Lilly et al. argue that brighter, $\sim 24$ mag galaxies are typically at $z=0.3$ and already exhibit a density excess.

Both of these differences are important for the observational investigation proposed here. If the high-redshift galaxies really are blue then their colour can be used to separate them from nearby galaxies ( $c f$. Tyson, Valdes \& Wenk 1990) and improve the signal-to-noise in the measured polarization. Furthermore, if the image shapes are as small as reported by Lilly et al., then it will be correspondingly harder to measure the individual image orientations. In particular a greater premium will be placed on observing under conditions of excellent seeing.

Magnification fluctuations are also predicted using our formalism. However, we have de-emphasized these in the present paper because we believe that their small amplitude (typically $\sim 0.03 \mathrm{mag}$ ) for CDM will be very difficult to disentangle from the effects of variable reddening. These fluctuations will be most apparent in galaxy counts down to a given limiting magnitude. If we use the counts per unit solid angle on the sky to define an angular density correlation function, $w(\boldsymbol{\theta})$, by

$\left\langle N N^{\prime}\right\rangle=\langle N\rangle^{2}[1+w(\theta)]$

and the source counts increase at a rate of $q \mathrm{mag}^{-1}$, then

$w(\theta)=(2.5 \log q)^{2}\left\langle\Delta M_{1} \Delta M_{2}\right\rangle=(2.5 \log q)^{2} C_{\mathrm{pp}}(\theta)$.

At the faintest magnitudes, $q \sim 2$.

We can use the positions of galaxies to infer the polarization. The shear field will not only shear the shapes of galaxies, it will also shear the distribution of galaxies on the sky. A spherical distribution of galaxies will thus become elongated and the twopoint correlation function of galaxies will acquire a quadrupole term, $w_{2}(\theta)=w(\theta) * C_{\mathrm{pp}}(\theta)$. This procedure has the advantage that it is not necessary to measure eccentricities and position angles for the galaxies. The measurements are thus affected much less by systematic effects. A drawback is that at the relevant distances, $z \sim 1, w(\theta)$ is already weak so we are looking for a very small effect. This procedure will be discussed in a future paper.

There is an important distinction that should be drawn between the observational investigation described here and several of the studies to which we have already referred. What we propose is to use the shear correlated over large angular scales to 'weigh' the large-scale structure. This involves measuring the shapes of tens of thousands of galaxies in $\sim 10-20$ arcmin fields. By determining the mean orientation, we will implicitly average over the intrinsic ellipticity distribution which may, for a variety of reasons, show some cosmological evolution.

It is possible that gravitational anisotropy in the source region might exert a subtle influence upon the intrinsic source orientation and a positive image polarization measurement might result (West 1989; West, Villumsen \& Dekel 1991). As the source galaxies are likely to be distributed over a large interval of comoving distance, uncorrelated regions will add stochastically. Intrinsic polarization should be distinguishable if a redshift indicator such as magnitude or colour can be found for the source galaxies. It should be emphasized that the treatment we have presented in this paper is restricted to an Einstein-de Sitter cosmology.

It is possible to use the modestly faint galaxies with magnitude $22 \leqslant m \leqslant 25$ to predict the image polarization, if they are located at redshifts $0.3 \leqslant z \leqslant 0.8$ where most of the distortion originates. Galaxy counts may provide a direct measure of the mass distribution at these intermediate redshifts. The density inhomogeneity associated with these galaxies can be computed for given models like CDM and different prescriptions for relating the galaxy magnitudes and colours to distance and used to predict the image polarization. This predicted image polarization can then be cross-correlated with the measured value. Unfortunately, this 
procedure will be more sensitive to small reddening variation than the measurement of ellipticity because it will be difficult to distinguish an apparent excess in the density of distorting galaxies from a negative fluctuation in the extinction.

We have argued that, if the faintest galaxies are really located at redshifts beyond $z=1$, and they can be adequately imaged, then random errors associated with counting statistics should be smaller than the anticipated signal. However, systematic errors are likely to be far more serious. There are several possible sources. First, differential refraction in the atmosphere will create elongated images. This effect can be minimized by using narrow band filters and working at high altitude. Secondly, similar effects inside the telescope itself, notably astigmatism, might contribute at the few per cent level. Thirdly, errors associated with guiding the telescope might create spurious elongation in right ascession. Although difficult to avoid, it is straightforward to test this by checking for any such correlation in the data. Fourthly, there is an intrinsic anisotropy in a gridded detector like a CCD. This can obviously be avoided by rotating the detector through multiples of $45^{\circ}$. Undoubtedly, the best method for quantifying all of these systematic effects is to compare the galaxy images with the point spread function measured using stars of similar colour.

An investigation like the one that we are proposing will require a large amount of telescope time. The galaxy counts to 29 mag of $3 \times 10^{5} \mathrm{deg}^{-2}$, reported by Tyson (1988) were obtained with six-hour integrations under conditions of excellent seeing using a 4-m telescope with a $3 \times 5 \operatorname{arcmin}^{2}$ field of view. (The photon counting rate is only $\sim 0.1 \mathrm{~s}^{-1}$ galaxy $^{-1}$.) This area of sky contains only $\sim 10^{3}$ galaxies and many nights would be necessary. Larger telescopes, like the Keck 10-m or the ESO VLT $16-\mathrm{m}$ telescope, will increase the rate of data acquisition, and a larger field of view would undoubtedly be beneficial. Tyson (private communication) reports the imminent availability of $50 \times 50$ arcmin CCDs which might acquire several hundred thousand galaxy images per night. (Note that observing over a wide field may increase the image distortion associated with the telescope.)

What might we learn from a positive detection of correlated ellipticity in galaxy images? In principle, this should be able to tell us the mass-to-light ratio on the largest scales of inhomogeneity yet probed. In practice, this will be quite sensitive to the assumed cosmology and somewhat sensitive to the assumed source redshift distribution. (We plan to address this in a future publication.) Although the large-scale structure so far observed is at too small a redshift to influence the image shapes seriously, it may be possible to probe areas of sky with unusually large induced ellipticity using pencil beam galaxy surveys (redshift surveys at intermediate distances and magnitude surveys at larger distance). It might then be possible to relate the distortion to specific inhomogeneities in the galaxy distribution. An alternative possibility is that high polarization will be associated with large excesses of quasar absorption lines.

A positive detection of correlated ellipticity would measure the mass distribution on the largest scale for which we have good local evidence of inhomogeneity. However, the most intriguing possibility of all, and the easiest result for which it can be believed that systematic observational errors are unimportant, is that no induced ellipticity will be found. This is what happened to measurements of microwave background fluctuations that were supposed to detect inhomogeneity on the last scattering surface. Such a contradiction between the direct observation of large-scale structure and the absence of associated image distortion would present a major challenge to cosmogony.

\section{ACKNOWLEDGMENTS}

This research was supported by the National Science Foundation under grants AST 86-15325, AST 89-15326 (RDB) and AST 89-21001 (JVV), the Carlsberg Foundation and the Danish Research Academy (ABS). Parts of the calculations were performed at the Ohio Supercomputer Center. We thank S. Cranmer and R. Emmering for assistance with the graphics. We are indebted to B. Fort and T. Tyson for advice and encouragement and the referee S. White for helpful suggestions which led to an improvement in the manuscript.

\section{REFERENCES}

Babul, A. \& Lee, M. H., 1990. Preprint.

Bahcall, N. A. \& Soneira, R. M., 1984. Astrophys. J., 277, 37.

Bardeen, J. M., Bond, J. R., Kaiser, N. \& Szalay, A. S., 1986. Astrophys. J., 304, 15 (BBKS).

Baron, E. J. \& White, S. D. M., 1987. Astrophys. J., 322, 585.

Birkenshaw, M., 1986. Quasars, IAU Symp. No. 119, p. 539, eds Swarup, G. \& Kapahi, V. K., Kluwer, Dordrecht.

Blandford, R. D., 1990. Q. J. R. astr. Soc., 31, 305.

Blandford, R. \& Jaroszyński, M., 1981. Astrophys. J., 246, 1.

Blandford, R. \& Narayan, R., 1986. Astrophys. J., 310, 568.

Broadhurst, T. J., Ellis, R. S., Koo, D. C. \& Szalay, A. S., 1990. Nature, 343, 726.

Davis, M., Efstathiou, G., Frenk, C. S. \& White, S. D. M., 1985. Astrophys. J., 292, 371.

Dyer, C. \& Oattes, L., 1988. Astrophys. J., 326, 50.

Efstathiou, G., Kaiser, N., Saunders, W., Lawrence, A., Rowan-Robinson, M., Ellis, R. S. \& Frenk, C. S., 1990. Mon. Not. R. astr. Soc., 247, 1P.

Efstathiou, G., Davis, M., Frenk, C. S. \& White, S. D. M., 1985. Astrophys. J. Suppl., 57, 241.

Fort, B., Prieur, L., Mathez, G., Mellier, Y. \& Soucail, G., 1988. Astr. Astrophys., 200, L17.

Geller, M. \& Huchra, J., 1989. Science, 246, 897.

Grossman, S. \& Narayan, R., 1988. Astrophys. J. Lett., 324, L37.

Guhathakurta, P., Tyson, J. A. \& Majewski, S., 1991. Astrophys. J. Lett., in press.

Gunn, J. E., 1967a. Astrophys. J., 147, 61.

Gunn, J. E., 1967b. Astrophys. J., 150, 737.

Jaroszyński, M., Park, C., Pasczyński, B. \& Gott, J., 1990. Astrophys. J., 365, 22. 
Kochanek, C., 1991. Mon. Not. R. astr. Soc., 371, 289.

Kristian, J., 1967. Astrophys. J., 147, 864.

Kristian, J. \& Sachs, R. K., 1967. Astrophys. J., 143, 379.

Lilly, S. J., Cowie, L. L. \& Gardner, J. P., 1991. Astrophys. J., 369, 79.

Linder, E., 1990. Mon. Not. R. astr. Soc., 243, 353.

Lynden-Bell, D., Faber, S. M., Burstein, D., Davies, R. L., Dressler, A., Terlevich, R. J. \& Wagner, G., 1988. Astrophys. J., $326,19$.

Lynds, C. \& Petrosian, V., 1989. Astrophys. J., 336, 1.

Maddox, S. J., Efstathiou, G., Sutherland, W. J. \& Loveday, J., 1990. Mon. Not. R. astr. Soc., 242, 43P.

Miralda-Escudé, J., 1991. Preprint.

Miralda-Escudé, J., 1990. Preprint.

Morse, P. M. \& Feshbach, H., 1953. Methods of Mathematical Physics, McGraw-Hill, New York.

Park, C., 1990. Mon. Not. R. astr. Soc., 242, 59p.

Peebles, P. J. E., 1980. The Large-Scale Structure of the Universe, Princeton University Press, Princeton.

Pelló-Descayre, R., Soucail, G., Sanahuja, B., Mathez, G. \& Ojero, E., 1988. Astr. Astrophys., 190, L11.

Penrose, R., 1966. Perspectives in Geometry and Relativity, ed. Hoffmann, B., Indiana University Press, Bloomington.

Sachs, R., 1961. Proc. R. Soc. Lond., A264, 309.

Saunders, W., Frenk, C., Rowan-Robinson, M., Efstathiou, G., Lawrence, A., Kaiser, N., Ellis, R., Crawford, J., Xia, X. \& Parry, I., 1991. Nature, $349,32$.

Soucail, G., Fort, B., Mellier, Y. \& Picat, J., 1987. Astr. Astrophys., 172, L14.

Soucail, G., Mellier, Y., Fort, B., Mathez, G. \& Cailloux, M., 1988. Astr. Astrophys., 191, L19.

Tomita, K. \& Watanabe, K., 1989. Prog. Theo. Phys., 82, 563.

Tyson, J. A., 1988. Astr. J., 96, 1.

Tyson, J. A. \& Seitzer, P., 1988. Astrophys. J., 335, 552.

Tyson, J. A., Valdes, F., Jarvis, J. \& Mills, A., 1984. Astrophys. J. Lett., 281, L59.

Tyson, J. A., Valdes, F. \& Wenk, R., 1990. Astrophys. J. Lett., 349, L1.

Valdes, F., Tyson, J. A. \& Jarvis, J. F., 1983. Astrophys. J., 271, 431.

Villumsen, J. V., 1989. Astrophys. J. Suppl., 71, 407.

Villumsen, J. V. \& Brainerd, T. G., 1990. Preprint.

Watanabe, K. \& Sasaki, M., 1990. Publs astr. Soc. Japan, 42, L33.

Webster, R. L., 1985. Mon. Not. R. astr. Soc., 213, 871.

West, M. J., 1989. Astrophys. J., 347, 610.

West, M. J., Villumsen, J. V. \& Dekel, A., 1991. Astrophys. J., 369, 287.

White, S. D. M., Frenk, C. S., Davis, M. \& Efstathiou, G., 1987. Astrophys. J., 313, 505.

\section{APPENDIX A: SURFACE POTENTIAL OF AN INCLINED UNIFORM DISC}

Consider a thin circular ring with surface density $\Sigma$, radius $r$ and thickness $d r$ inclined at an angle $i$ to the line-of-sight so that the axis ratio of the projected ring is $\cos (i)$ and the projected surface density is $\Sigma \sec (i)$. We know from potential theory (e.g. Morse $\&$ Feshbach 1953) that the interior surface potential $d \Phi^{(2)}$ is constant everywhere and the exterior equipotential curves form a family of confocal ellipses $u=$ constant, where the curvilinear coordinates $u, v$ are related to Cartesian coordinates $x_{1}$, $x_{2}$ through

$x_{1}=f u v$
$x_{2}=f\left(u^{2}-1\right)^{1 / 2}\left(1-v^{2}\right)^{1 / 2}$$\quad\left\{\begin{array}{l}1<u, \\ 0<v<1 .\end{array}\right.$

The semi-major axis of these ellipses are $u f$ and the eccentricities are $e=1 / u$. Solving Laplace's equation in terms of these coordinates, we obtain

$$
\begin{array}{rlrl}
d \Phi^{(2)} & =4 \pi \Sigma r d r \cosh ^{-1} u, & u \geq \operatorname{cosec} i \\
& =4 \pi \Sigma r d r \ln [\cot (i / 2)] \quad u \leq \operatorname{cosec} i .
\end{array}
$$

Now let us assemble the potential for a complete disc by decomposing it into annular rings and integrating over them.

$\Phi^{(2)}\left(x_{1}, x_{2}\right)=4 \pi\left[\int_{0}^{\left(x_{1}^{2}+x_{2}^{2} \sec ^{2} i\right)^{1 / 2}} \Sigma(r) r d r \cosh ^{-1}(u)+\int_{\left(x_{1}^{2}+x_{2}^{2} \sec ^{2} i\right)^{1 / 2}}^{\infty} \underset{(r)}{ } \Sigma d r \ln [\cot (i / 2)]\right]$,

where $u(r)$ solves

$\frac{x_{1}^{2}}{u^{2}}+\frac{x_{2}^{2}}{u^{2}-1}=r^{2} \sin ^{2} i$.

The two-dimensional gravity can be computed by differentiating equation (A111). After some calculation, we obtain

$$
g(\boldsymbol{x})=\left|\nabla \Phi^{(2)}\right|=2 \pi \operatorname{cosec}^{2} i d u^{2} \Sigma\left(u^{2}-1\right)^{-1} u^{-2}\left[\left(\frac{u^{2}-1}{u^{2}}\right) x_{1}^{2}+\left(\frac{u^{2}}{u^{2}-1}\right) x_{2}^{2}\right]^{1 / 2}
$$

directed normal to the equipotential surfaces. 
Now let us simplify by assuming that the surface density, $\Sigma$, is constant within a radius $R$. Resolving $g$ into its Cartesian components, we obtain

$g_{1}=-\frac{4 \pi \Sigma x_{1}}{1+\cos i}$

and

$g_{2}=-\frac{4 \pi \Sigma x_{2}}{\cos i(1+\cos i)}$

for impact parameters that intercept the disc, as may be verified by elementary means (e.g. Birkenshaw 1986).

For rays that pass outside the disc, the integral over $u$ must be carried out to a maximum value of $u_{\mathrm{m}}$ satisfying

$\frac{x_{1}^{2}}{u_{\mathrm{m}}^{2}}+\frac{x_{2}^{2}}{u_{\mathrm{m}}^{2}-1}=R^{2} \sin ^{2} i$

Changing variables to $\zeta=u^{-2}\left(\right.$ and $\left.\zeta_{\mathrm{m}}=u_{\mathrm{m}}^{-2}\right)$, we obtain

$g_{1}=-4 \pi \Sigma \operatorname{cosec}^{2} i x_{1}\left[1-\left(1-\zeta_{\mathrm{m}}\right)^{1 / 2}\right]$

$g_{2}=4 \pi \Sigma \operatorname{cosec}^{2} i x_{2}\left[1-\left(1-\xi_{m}\right)^{-1 / 2}\right]$,

where

$\zeta_{\mathrm{m}}=\frac{\left(x_{1}^{2}+x_{2}^{2}+R^{2} \sin ^{2} i\right)-\left[\left(x_{1}^{2}+x_{2}^{2}+R^{2} \sin ^{2} i\right)^{2}-4 x_{1}^{2} R^{2} \sin ^{2} i\right]^{1 / 2}}{2 x_{1}^{2}}$.

We need the gravity gradients to compute the shear. We obtain

$\frac{\partial g_{1}}{\partial x_{1}}=\cos i \frac{\partial g_{2}}{\partial x_{2}}=-\frac{4 \pi \Sigma}{1+\cos i}$

$\frac{\partial g_{1}}{\partial x_{2}}=\frac{\partial g_{2}}{\partial x_{1}}=0$

for rays passing through the disc. For rays passing outside the disc,

$\frac{\partial g_{1}}{\partial x_{1}}=-\frac{\partial g_{2}}{\partial x_{2}}=4 \pi \Sigma \operatorname{cosec}^{2} i \frac{\left[1-\left(1-\xi_{\mathrm{m}}\right)^{1 / 2}\right]\left[\left(1-\xi_{\mathrm{m}}\right)^{3 / 2} x_{1}^{2}-x_{2}^{2}\right]}{\left(1-\zeta_{\mathrm{m}}\right)^{2} x_{1}^{2}+x_{2}^{2}}$

and

$\frac{\partial g_{2}}{\partial x_{1}}=\frac{\partial g_{1}}{\partial x_{2}}=\frac{4 \pi \Sigma \operatorname{cosec}^{2} i x_{1} x_{2} \zeta_{\mathrm{m}}\left(1-\zeta_{\mathrm{m}}\right)^{1 / 2}}{\left(1-\zeta_{\mathrm{m}}\right)^{2} x_{1}^{2}+x_{2}^{2}}$. 